\title{
Effect of Ice Shape Fidelity on Swept-Wing Aerodynamic Performance
}

\author{
Stephanie C. Camello ${ }^{1}$ and Michael B. Bragg ${ }^{2}$, \\ University of Washington, Seattle, WA, 98115, USA \\ Andy P. Broeren ${ }^{3}$ \\ NASA John H. Glenn Research Center, Cleveland, OH, 44135, USA \\ Christopher W. Lum ${ }^{4}$ \\ University of Washington, Seattle, WA, 98115, USA \\ Brian S. Woodard ${ }^{5}$ \\ University of Illinois at Urbana-Champaign, Urbana, IL, 61801, USA \\ and \\ Sam Lee ${ }^{6}$ \\ Vantage Partners, LLC, Cleveland, OH, 44135, USA
}

Low-Reynolds number testing was conducted at the $7 \mathrm{ft} \times 10 \mathrm{ft}$ Walter $H$. Beech Memorial Wind Tunnel at Wichita State University to study the aerodynamic effects of ice shapes on a swept wing. A total of 17 ice shape configurations of varying geometric detail were tested. Simplified versions of an ice shape may help improve current ice accretion simulation methods and therefore aircraft design, certification, and testing. For each configuration, surface pressure, force balance, and fluorescent mini-tuft data were collected and for a selected subset of configurations oil-flow visualization and wake survey data were collected. A comparison of two ice shape geometries and two configurations with simplified geometric detail for each ice shape geometry is presented in this paper.

\section{Nomenclature}

NASA $\quad=$ National Aeronautics and Aerospace Administration

$F A A=$ Federal Aviation Administration

\footnotetext{
${ }^{1}$ Research Assistant, William E. Boeing Department of Aeronautics and Astronautics, University of Washington; Guggenheim Hall Room 211, Box 352400, Seattle, WA 98195-2400, Member AIAA.

2 Dean, College of Engineering and Professor, William E. Boeing Department of Aeronautics and Astronautics, University of Washington; Loew Hall Room 371, Box 352180, Seattle, WA 98195-2180, Fellow AIAA.

${ }^{3}$ Research Aerospace Engineer, Icing Branch, 21000 Brookpark Rd., MS 11-2, Associate Fellow AIAA.

${ }^{4}$ Research Assistant Professor, William E. Boeing Department of Aeronautics and Astronautics, University of Washington; Guggenheim Hall Room 211, Box 352400, Seattle, WA 98195-2400, Member AIAA.

${ }^{5}$ Research Associate, Dept. of Aerospace Engineering, 306 Talbot Lab, 104 S. Wright St., Member AIAA.

${ }^{6}$ Engineer IV, Icing Branch, 21000 Brookpark Rd., MS 11-2, Senior Member AIAA.
} 


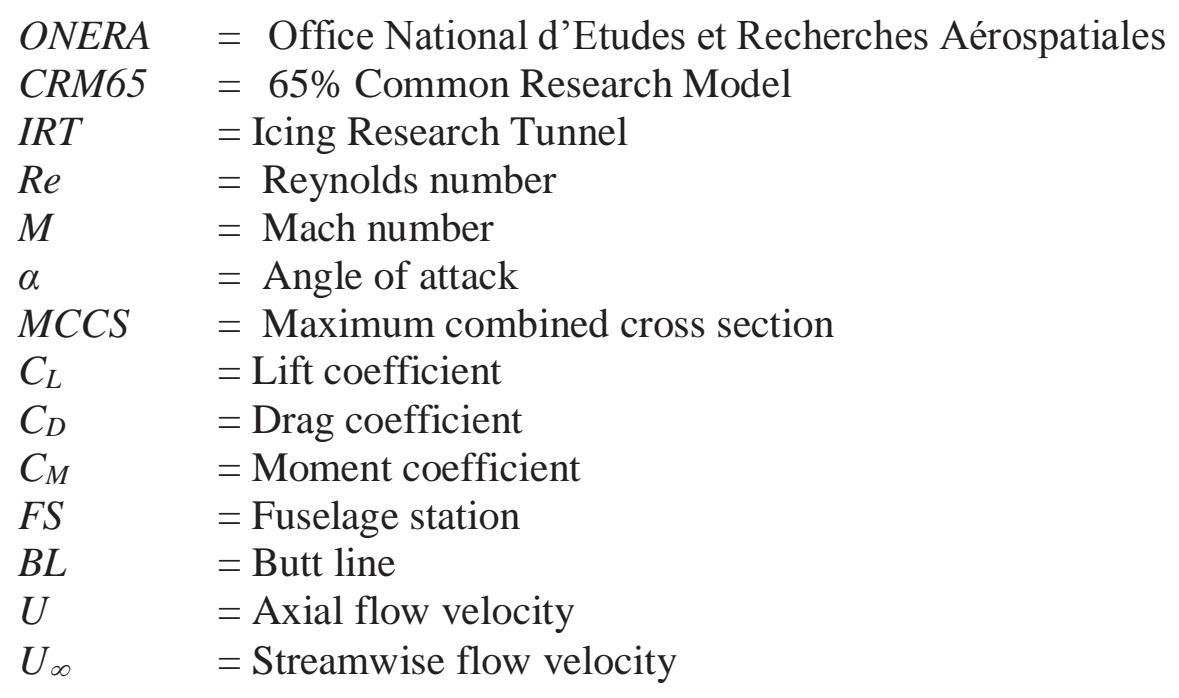

\section{Introduction}

The aerodynamic effect of ice accretion on swept wings is complex compared to the more widely studied airfoil case and is currently not commonly understood nor presented in the public literature on aircraft icing. A better understanding of these effects is important in the design and certification of transport jet aircraft. Currently a collaborative research effort is underway including the National Aeronautics and Space Administration (NASA), the Federal Aviation Administration (FAA), the Office National d'Etudes et Recherches Aérospatiales (ONERA), the University of Washington (UW), the University of Illinois at Urbana-Champaign (UIUC), the University of Virginia (UVA), and Boeing on swept-wing icing. This research program is expanding our understanding, and improving experimental and computational tools, of ice accretion and the aerodynamic effects of ice on swept wings. ${ }^{1}$ This paper represents the preliminary analysis of some of the early research program data on the aerodynamics of a swept wing with simulated ice accretion.

To understand this work, it is useful to briefly review the extensive study of ice accretion aerodynamics on airfoils that preceded it. In the early 2000s a similar research program to the current effort was conducted on 2D airfoils. ${ }^{2}$ This project culminated many years of research by NASA and others on iced airfoil aerodynamics. From this experience, categories of ice shapes were developed ${ }^{3}$ based on their aerodynamic effect and primary flowfield features. While some similarities exist, this differs from the more common classification of ice shapes by their accretion mechanisms (rime and glaze, for example). Four ice shape categories were identified: ice 
roughness, streamwise, horn, and spanwise-ridge ice. Each of these categories have fundamentally different iced airfoil aerodynamics resulting from different flowfield physics. ${ }^{3}$

A research program was designed and conducted to generate these ice accretions in the NASA IRT, simulate the shapes at full and subscale, and evaluate the aerodynamic performance of simulated shapes of varying degrees of fidelity. ${ }^{4,5}$ Ice accretion data were obtained on a six-foot chord NACA 23012 airfoil model in the icing tunnel; cast and molding techniques were used to document the accretions and provide the high-fidelity shapes for aerodynamic wind tunnel testing. These high-fidelity cast ice shapes were tested on a full-scale NACA 23012 model in the ONERA F1 pressurized wind tunnel to provide the baseline data over a range of Reynolds numbers and Mach numbers. Large lift and drag penalties were observed as a result of the cast ice accretions with the largest penalty in maximum lift being 52 percent from the spanwise-ridge shape. The roughness simulations and the streamwise ice produced much smaller, but still very significant performance penalties with maximum lift reduced on the order of 33 percent. Drag increases over the angle of attack range tested were also significant. These tests confirmed and reinforced earlier findings from testing in the NASA Low-Turbulence Pressure Tunnel, LTPT ${ }^{6}$, that while Reynolds number and Mach number effects may be significant on the clean airfoil, they are generally small on the iced airfoil. Testing in the F1 tunnel on this airfoil model was performed over the Reynolds number range from $4.6 \times 10^{6}$ to $16 \times 10^{6}$ and a Mach number range from 0.10 to 0.28.

Low-Reynolds number testing $\left(\operatorname{Re}=1.8 \times 10^{6}\right)$ on a subscale NACA 23012 airfoil model was then used to evaluate the effect of ice accretion fidelity on measured aerodynamic performance. ${ }^{7,8,9}$ While the cast shapes tested in the F1 tunnel provided the relatively complete three-dimensional roughness profile and spanwise variation of the ice shapes, at the time digital scanning and rapid prototyping methods were not sufficiently developed to be used in that test program to capture the three-dimensional ice accretions. Thus, no high-fidelity ice shapes for the subscale model were tested at low-Reynolds numbers. Busch ${ }^{7}$ tested three levels of low-fidelity shapes. These included simple geometric shapes, two-dimensional extrusions based on a measured ice cross section, and shapes that included simple representations of the observed spanwise variation in the measured ice accretion. A good representation of iced-airfoil performance was observed on the low-fidelity shapes at low-Reynolds number as compared to the cast shapes at high-Reynolds number. Busch ${ }^{7}$ carefully documents and discusses these results by ice shape category and level of fidelity. Overall this research program provided a comprehensive look at airfoil ice accretion and full and subscale 
performance including the effects of ice shape category, simulation fidelity, and Reynolds number and Mach number effects.

The current swept-wing icing study builds on the previous 2D airfoil program, but presents different, and in many ways, more difficult technical challenges, while benefiting from recent advances in CFD and experimental tools. ${ }^{1}$ As in the previous study, classifications of swept wing ice accretion have been developed and are guiding and organizing the present aerodynamic study. ${ }^{10}$ The baseline configuration for this research is a $65 \%$ scale version of the Common Research Model (CRM65). The CRM65 is representative of a typical commercial transport jet as it is comparable in size to a Boeing 757. It was chosen due to the public availability of the geometric and aerodynamic data associated with this model. Simulation tools were used to determine that the ice accretions across the entire span could be sufficiently captured using three models. Due to the large chord length of the CRM65, the models had to be truncated. Hybrid models were designed to keep the full-scale leading edge and leading-edge aerodynamics with significantly reduced chord length. Fujiwara et. al ${ }^{11,12,13}$ describe this process in detail.

NASA IRT tests produced the ice accretions ${ }^{14}$ while laser scanning produced detailed digital presentations and documented the accretions for ice accretion code comparisons as well as aerodynamic testing. Methods were developed to morph these shapes into 3D fullspan ice shapes and wind-tunnel model leading edges were built using rapid prototyping methods. ${ }^{15}$ The program has aerodynamic objectives to understand the importance of ice shape fidelity as well as Reynolds number and Mach number on aerodynamic performance. Therefore, the artificial ice shapes generated for this work are considered representative of realistic, full-scale, in-flight ice that may accrete on a commercial, swept-wing transport airplane. Unlike the previous airfoil program, very little of these types of data are currently available for swept-wing icing. Aerodynamic testing is being conducted in both a low-Reynolds number atmospheric wind tunnel facility on an $8.9 \%$ scale model of the semispan swept wing and in the ONERA F1 pressure tunnel high-Reynolds number facility on a $13.3 \%$ scale wing model as part of this research program.

The purpose of this paper is to present preliminary results of low-Reynolds number wind tunnel testing for ice shapes of varying fidelity and compare the aerodynamics of the swept-wing model for the different ice shapes. Broeren et al. ${ }^{16}$ in an earlier paper described in detail the development and implementation of experimental setup, supporting CFD, and initial clean wing results for the low-Reynolds number testing. The methods for creating rapid-prototyped low- and high-fidelity 
artificial ice shapes for aerodynamic wind tunnel testing is described briefly here and in more detail in Camello et. al. ${ }^{15,17}$ The high-Reynolds number results and a more comprehensive analysis of results will be presented as these become available as the research program continues.

\section{Wind-Tunnel Facility, Model, and Experimental Methods}

Low-Reynolds number wind tunnel testing was conducted at the Walter H. Beech Memorial Wind Tunnel at Wichita State University. It is an atmospheric, closed-return type, subsonic wind tunnel with a $7 \mathrm{ft} \times 10 \mathrm{ft}$ test section. A 6-component pyramidal style force balance was used to collect the lift, drag, and pitching moment on the model, and an Esterline miniature electronic pressure scanning module (ESP-32HD) was used to acquire surface pressure measurements. ${ }^{18}$ Wind tunnel wall corrections were applied to all force balance data collected using the method by Pope et. $\mathrm{al}^{19}$ and an experimental uncertainty analysis was performed and is presented by Diebold et. al. ${ }^{20}$

An $8.9 \%$ scale model of the baseline configuration, the CRM65, was designed and manufactured for this research project. The CRM65 is a $65 \%$ scale version of the Common Research Model which was designed by NASA and the Boeing Company for CFD validation exercises. ${ }^{20,21}$ To test artificial ice shapes efficiently, the subscale model was designed with a removable leading edge that could be interchanged with rapid-prototyped artificial ice shapes. A summary of the swept-wing model parameters is summarized in Table 1 and the removable leading edge geometry is shown in Figure 1.

Table 1: Parameters of the $8.9 \%$ scale model of the CRM65. ${ }^{20}$

\begin{tabular}{lc}
\hline \multicolumn{1}{c}{ Wing Parameter } & Value \\
\hline Span & $5.00 \mathrm{ft}(60.0 \mathrm{in})$ \\
MAC & $1.39 \mathrm{ft}(16.67 \mathrm{in})$ \\
Area & $6.01 \mathrm{ft}^{2}\left(865.3 \mathrm{in}^{2}\right)$ \\
Volume & $0.617 \mathrm{ft} 3\left(1069 \mathrm{in}^{3}\right)$ \\
Aspect ratio & 8.30 \\
Taper ratio & 0.23 \\
Root chord & $2.25 \mathrm{ft}(27.0 \mathrm{in})$ \\
Tip chord & $0.52 \mathrm{ft}(6.20 \mathrm{in})$ \\
Root $\alpha$ & $4.40^{\circ}$ \\
Tip $\alpha$ & $-3.80^{\circ}$ \\
1/4-chord sweep angle & $35.0^{\circ}$ \\
Leading edge sweep angle & $37.2^{\circ}$ \\
\hline \hline
\end{tabular}




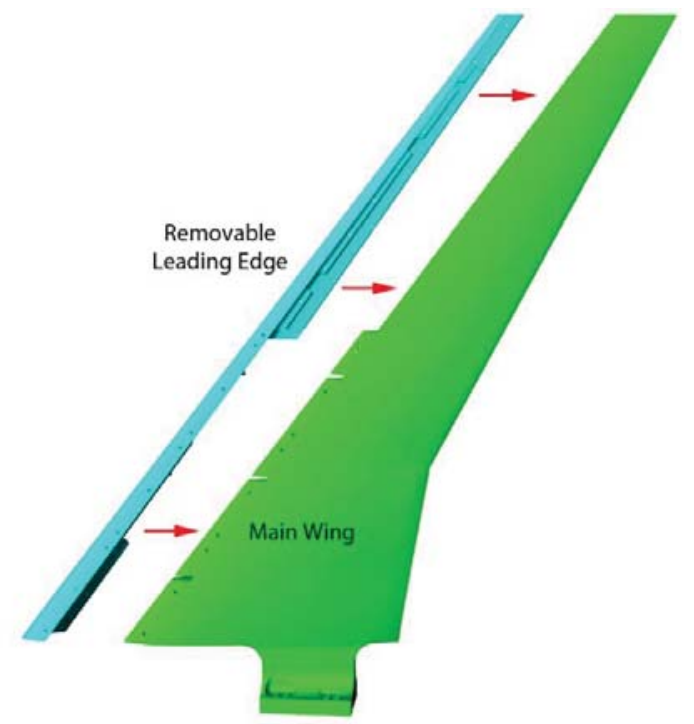

Figure 1: Removable leading edge feature on the 8.9\% scale CRM65 model. ${ }^{20}$

Full-span, high-fidelity, artificial ice shapes were created for aerodynamic testing using an interpolation and extrapolation method from a limited set of experimental ice accretions. The details of this process are presented in Camello et. al. ${ }^{15}$ The experimental ice accretion data were obtained from the NASA IRT using hybrid models, which contain a full-scale leading edge with a truncated aft section, of three spanwise locations (Inboard, Midspan, Outboard) of the CRM65. ${ }^{11,12,13}$ The full-scale leading edge was maintained to reduce or eliminate the icing scaling required when the entire wing geometry is scaled. A summary of the IRT cloud conditions chosen based upon airplane holding scenarios consistent with the Code of Federal Regulations (CFR) Title 14, Part 25, Appendix C Continuous Maximum icing is shown in Table 2. Broeren et. $\mathrm{al}^{14}$ further describe these reference and scale conditions for the IRT test campaigns that were used to generate representative, full-scale ice accretions. All the ice shape conditions besides the Streamwise condition produced ice shape geometries that fall under the horn ice category each with varying scalloped features. An example of the maximum combined cross sections (MCCS) of each case is shown in Figure 2 and detailed photographs of the ice accretions listed in Table 2 can be found in Camello et al. ${ }^{15}$ The MCCS were created by placing a series of cross sections taken along the span of the ice accretion geometry in a $2 \mathrm{D}$ plane and tracing the outer-most line to create a 2D representation of the ice accretion. 
Table 2: Summary of IRT cloud conditions. Adapted from Camello et al. ${ }^{15}$

\begin{tabular}{lcccccc}
\hline \hline Ice Shape Geometry & $\begin{array}{c}\mathbf{T}_{\mathbf{0}} \\
\left({ }^{\circ} \mathbf{C}\right)\end{array}$ & $\begin{array}{c}\mathbf{V} \\
(\mathbf{k t s})\end{array}$ & $\begin{array}{c}\text { MVD } \\
(\boldsymbol{\mu m})\end{array}$ & $\begin{array}{c}\text { LWC } \\
\left(\mathbf{g} / \mathbf{m}^{3}\right)\end{array}$ & $\begin{array}{c}\boldsymbol{\alpha} \\
\left.\mathbf{(}^{\circ}\right)\end{array}$ & $\begin{array}{c}\mathbf{t} \\
(\mathbf{m i n})\end{array}$ \\
\hline Venetian Blind & -3.8 & 130 & 25 & 1.0 & 3.7 & 29 \\
Maximum Scallop & -6.3 & 130 & 25 & 1.0 & 3.7 & 29 \\
Small Gap Scallop & -8.7 & 130 & 25 & 1.0 & 3.7 & 29 \\
Incomplete Scallop & -11.2 & 130 & 25 & 1.0 & 3.7 & 29 \\
Streamwise & -17.9 & 130 & 25 & 0.6 & 3.7 & 23 \\
Maximum Scallop Low $\alpha$ & -6.3 & 130 & 25 & 1.0 & 2.1 & 29 \\
WB33 & -3.1 & 130 & 25 & 0.9 & 3.7 & 49 \\
\hline \hline
\end{tabular}
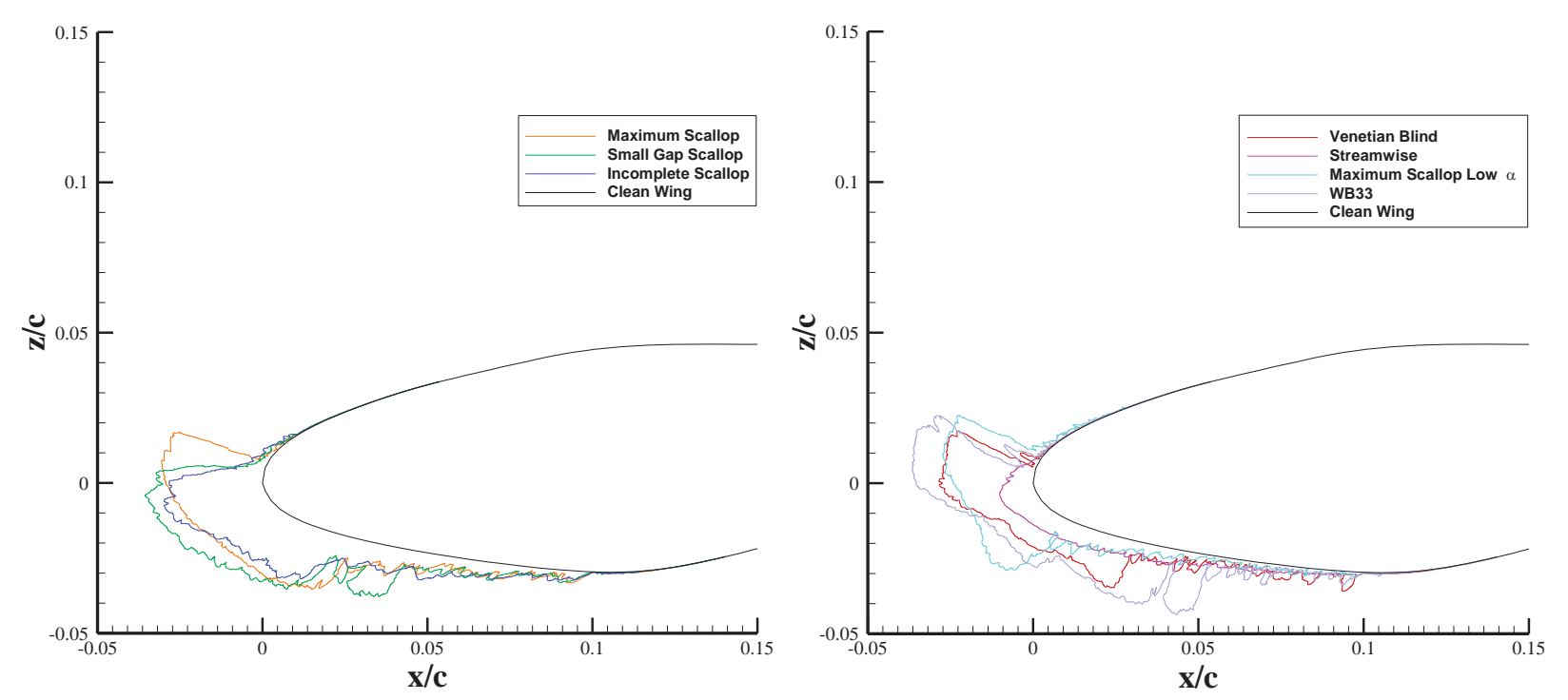

Figure 2: MCCS of the ice accretion on the Midspan location hybrid model.

Low-fidelity 2D smooth artificial ice shapes were created for aerodynamic testing by lofting through a series of simplified 2D cuts created from the geometry of a high-fidelity ice shape. This is one of numerous methods to create a low-fidelity ice shape and resulted in a geometry based on the largest local scallop. Other methods of creating low-fidelity ice shapes may result in different geometries with different aerodynamic properties. Ice shape roughness was simulated using 60grit silicon carbide applied to the low-fidelity 2D smooth ice shapes using double sided tape. This grit size was chosen based on guidance in AC25-25A $\mathrm{A}^{22}$ which recommended a $3 \mathrm{~mm}$ roughness height for the full-scale CRM65. The height was scaled to approximately $8.9 \%$ of the $3 \mathrm{~mm}$ to obtain the 60-grit size for the scaled model for low-Reynolds number wind tunnel testing. Both the low- and high-fidelity artificial ice shapes were merged with the geometry of the removable leading edge of the $8.9 \%$ scale CRM65 model and rapid prototyped using Somos $\mathrm{NeXt}^{23}$ material 
due to its moisture resistance and thermal properties. Examples of a high-fidelity, 2D smooth, and 2D smooth + grit artificial ice shape are shown in Figure 3 and the 17 different ice shape configurations created for low-Reynolds number aerodynamic testing are summarized in Table 3.
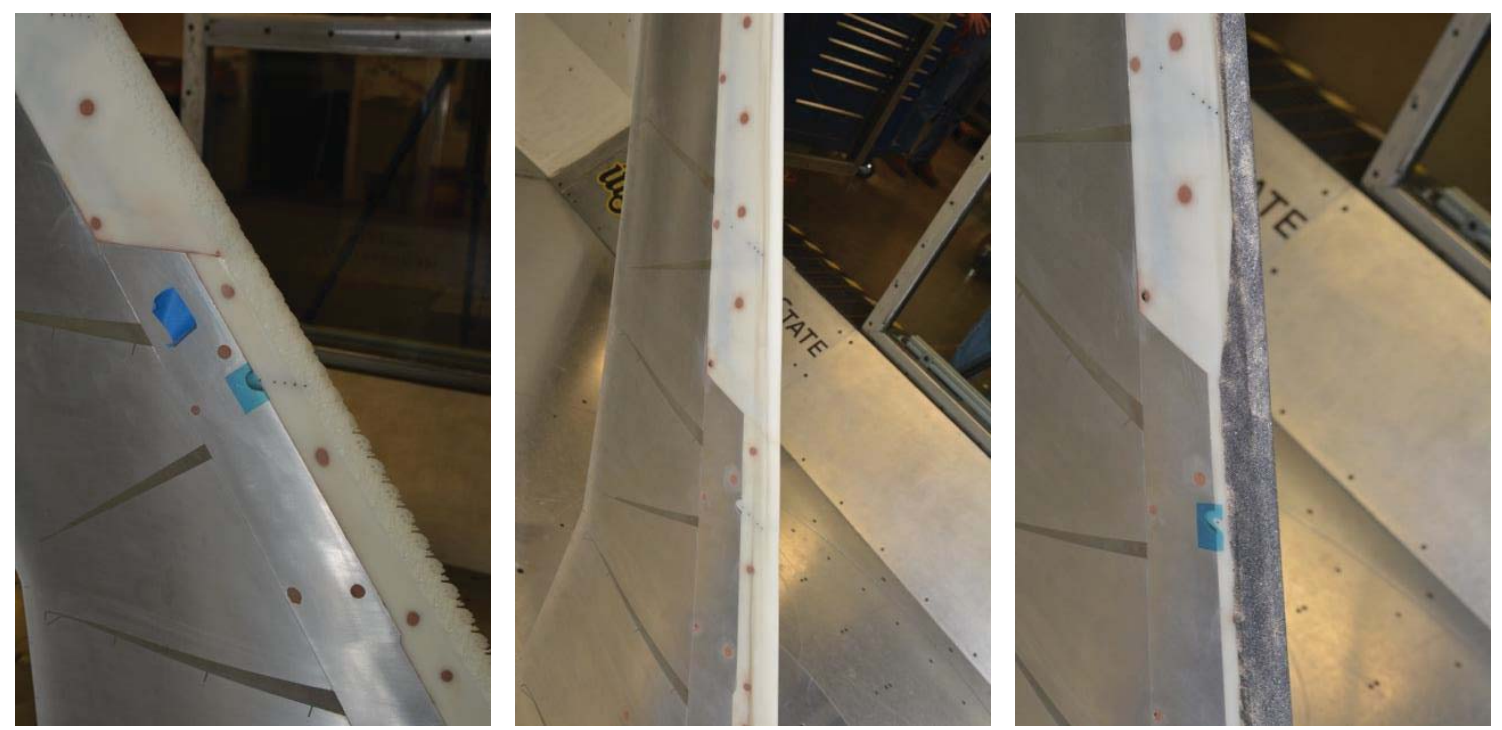

Figure 3: Shown left to right - High fidelity, 2D smooth, and 2D smooth + grit artificial ice shapes installed on the $8.9 \%$ scale CRM wing.

Table 3: Summary of ice shape configurations used for low-RE wind tunnel testing. ${ }^{17}$

\begin{tabular}{ccc}
\hline \hline Ice Shape ID & Ice shape geometry & Fidelity \\
\hline 1.1 .2 & Maximum Scallop & High-fidelity \\
1.2 .1 & “ & 2D smooth \\
1.2 .2 & Small Gap Scallop & 2D smooth + grit \\
3.1 & “ & High-fidelity \\
3.2 .1 & " & 2D smooth \\
3.2 .2 & “enetian Blind & 2D smooth + grit \\
2.1 & “ & High-fidelity \\
2.2 .1 & Maximum Scallop Low $\alpha$ & 2D smooth \\
2.2 .2 & “ & 2D smooth + grit \\
5.1 & “ & High fidelity \\
5.2 .1 & Streamwise & 2D smooth \\
5.2 .2 & “ & 2D smooth + grit \\
6.1 .2 & “ & High-fidelity \\
6.2 .1 & WB33 & 2D smooth \\
6.2 .2 & Incomplete Scallop & 2D smooth + grit \\
10.1 & High-fidelity \\
4.1 & & High-fidelity \\
\hline \hline
\end{tabular}

Force balance, surface pressure, fluorescent mini-tuft, surface oil-flow visualization, and wake survey $^{24}$ data were collected during aerodynamic testing. Force balance and surface pressure 
measurements were acquired at Reynolds numbers of $0.8 \times 10^{6}, 1.6 \times 10^{6}$, and $2.4 \times 10^{6}$ which corresponded to freestream Mach numbers of 0.09, 0.18, and 0.27. The model angle of attack was swept from $-6^{\circ}$ to $16^{\circ}$. Surface pressure data were used to create pressure contour plots based upon linear interpolation between the individual pressure taps (shown as small circles on the plots) on the upper surface of the wing. Fluorescent mini-tuft visualization was conducted using 0.0019inch diameter fluorescent monofilament pieces attached to the swept-wing model in streamwise rows. Images of the fluorescent mini-tuft visualization were captured for an angle of attack sweep at the same time as the force balance and surface pressure data. Surface oil-flow visualization was conducted for a subset of conditions by applying a mixture of mineral oil and fluorescent dye to black contact paper on the surface of the model. Images of the surface oil-flow visualization were captured after the tunnel was run at a predetermined speed and set model angle of attack for two minutes from fan on to fan off. Five-hole pressure probe wake survey data were collected for a subset of the ice shape configurations and the results are presented in Lum et al. ${ }^{24}$ The experimental methods used for this low-Reynolds number aerodynamic testing is described in more detail in Diebold et. al. ${ }^{20}$

\section{Results and Discussion}

\section{A. Clean Wing Flowfield}

The flowfield for the clean wing as stall occurred is shown for $\operatorname{Re}=1.6 \times 10^{6}$ and $\mathrm{M}=0.18$ as pressure contour plots, oil-flow visualization, and fluorescent mini-tuft visualization in Figure 4 Figure 6. Figure 4 shows the fluorescent mini-tuft and oil-flow visualization for the clean wing at $\alpha=11.1^{\circ}$. A leading-edge vortex that separated from the wing surface near the wing tip was observed in both flow visualization techniques. Spanwise flow near the trailing edge of the wing was most visible in the fluorescent mini-tuft visualization. 

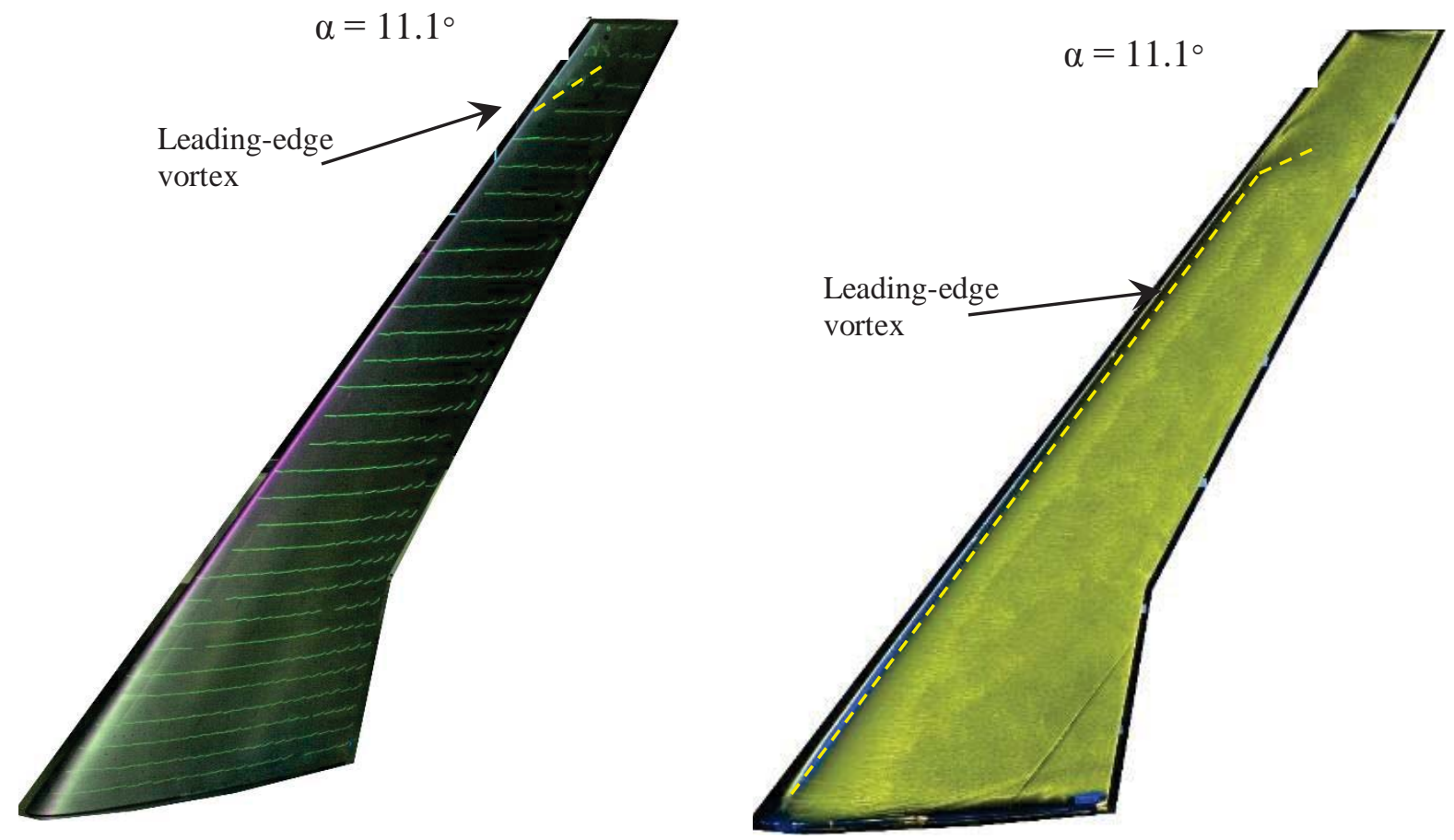

Figure 4: Comparison of mini-tuft (left) and surface-oil (right) flow visualization on upper surface of the clean wing configuration at $\alpha=11.1^{\circ}$ at $R e=1.6 \times 10^{6}$ and $M=0.18 .{ }^{16}$

Figure 5 and Figure 6 show the pressure contour plots at $\alpha=11.1^{\circ}, 13.6^{\circ}, 14.1^{\circ}$ and fluorescent mini-tuft visualization for the clean wing model at $\alpha=13.6^{\circ}, 14.1^{\circ}$, respectively. As the angle of attack was increased to $13.6^{\circ}$ the leading-edge suction increased which indicated that the vortex was growing stronger. Figure 5 shows that the leading-edge vortex separating from the surface of the model at $\alpha=13.6^{\circ}$ between the $\mathrm{y}=36 \mathrm{in}$. and $\mathrm{y}=45 \mathrm{in}$. locations which was reflected in the fluorescent mini-tuft data in Figure 6 with spanwise oriented tufts outboard of the 55\% span location. Although the location of separation of the leading-edge vortex may seem clear in Figure 5 , the grid of the surface pressure data collected from the surface pressure taps during testing was sparser than the grid of the interpolated surface pressures presented in Figure 5 and the following pressure contour plots. The high level of suction near the leading edge of the model was not maintained as the angle of attack was increased to $\alpha=14.1^{\circ}$ as shown in Figure 5 using the surface pressures. Fluorescent mini-tuft visualization in Figure 6 showed reversed flow across the outboard $80 \%$ of the wing surface. Further analysis of the clean wing configuration is described in Broeren et. al. ${ }^{16}$ 

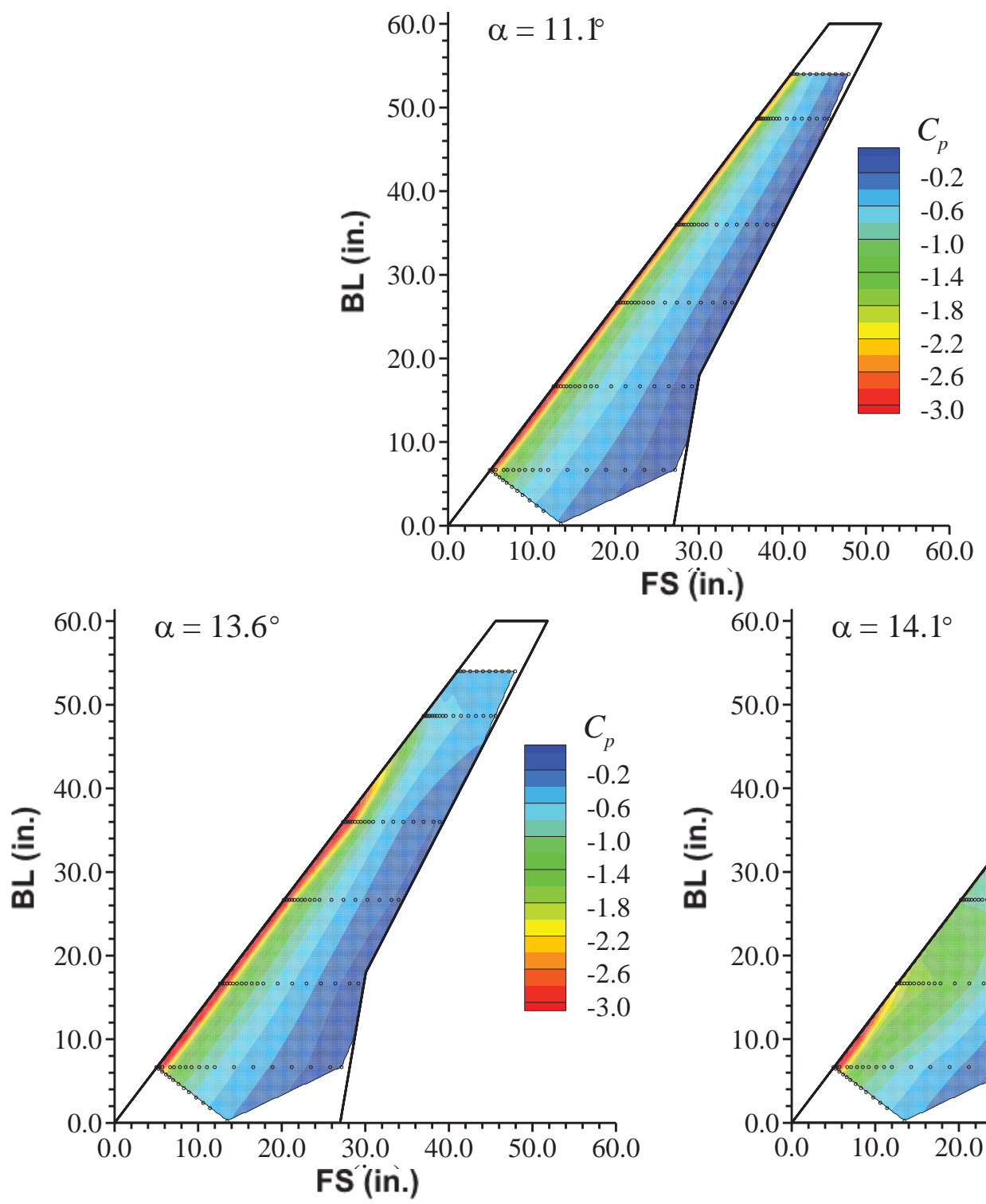

FS (in.)

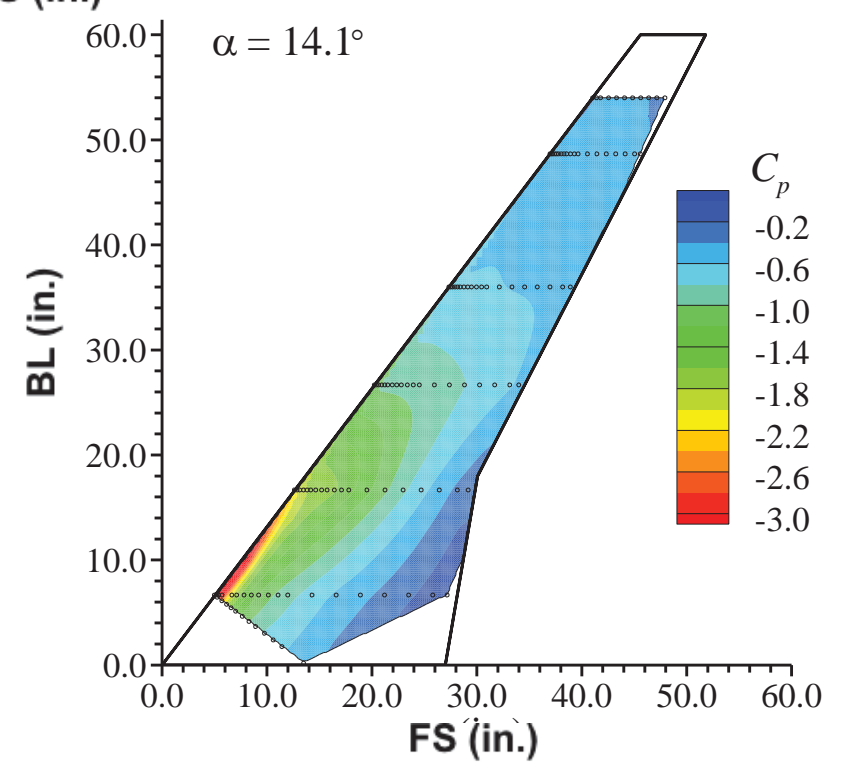

Figure 5: Contours of upper surface pressure of the clean wing configuration for $\alpha=13.6^{\circ}$ and $14.1^{\circ}$ at $R e=1.6 \times 10^{6}$ and $M=0.18 .{ }^{16}$ 

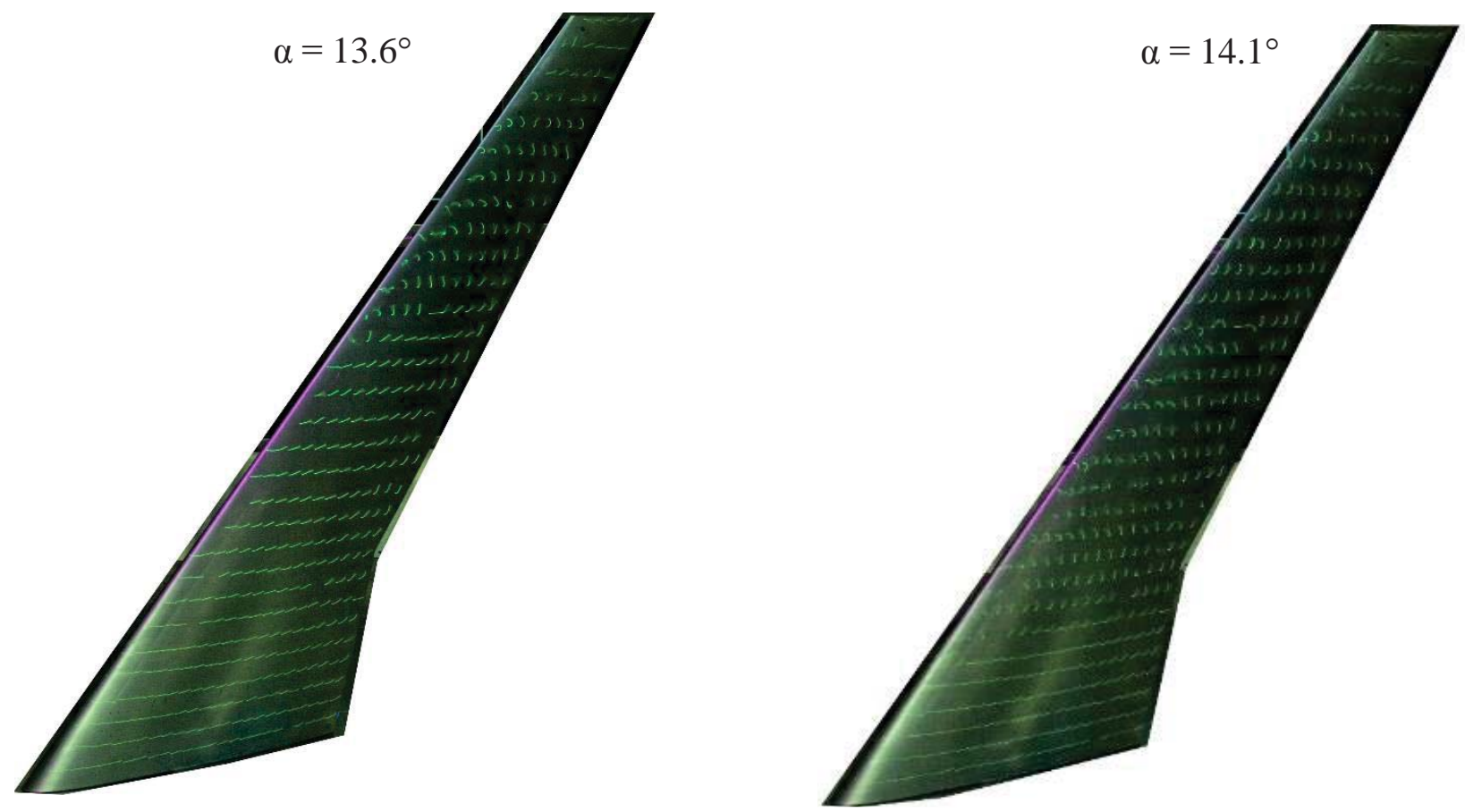

Figure 6: Mini-tuft flow visualization on upper surface for the clean wing configuration at $\alpha=$ $13.6^{\circ}$ and $14.1^{\circ}$ at $\operatorname{Re}=1.6 \times 10^{6}$ and $M=0.18 .^{16}$

\section{B. Fidelity comparison for all ice shape configurations}

A comparison of the aerodynamic data of the high-fidelity ice shape configurations and the clean wing data is shown in Figure 7. The coefficient of drag plot is shown from at $\alpha=-6.0^{\circ}$ to $6.0^{\circ}$ to emphasize the differences in aerodynamic performance between the ice shape geometries. The data showed that all the high-fidelity scalloped ice shapes produced extremely similar aerodynamic results while the Streamwise ice shape resulted in force balance data that was the most similar to the clean wing force balance data. 

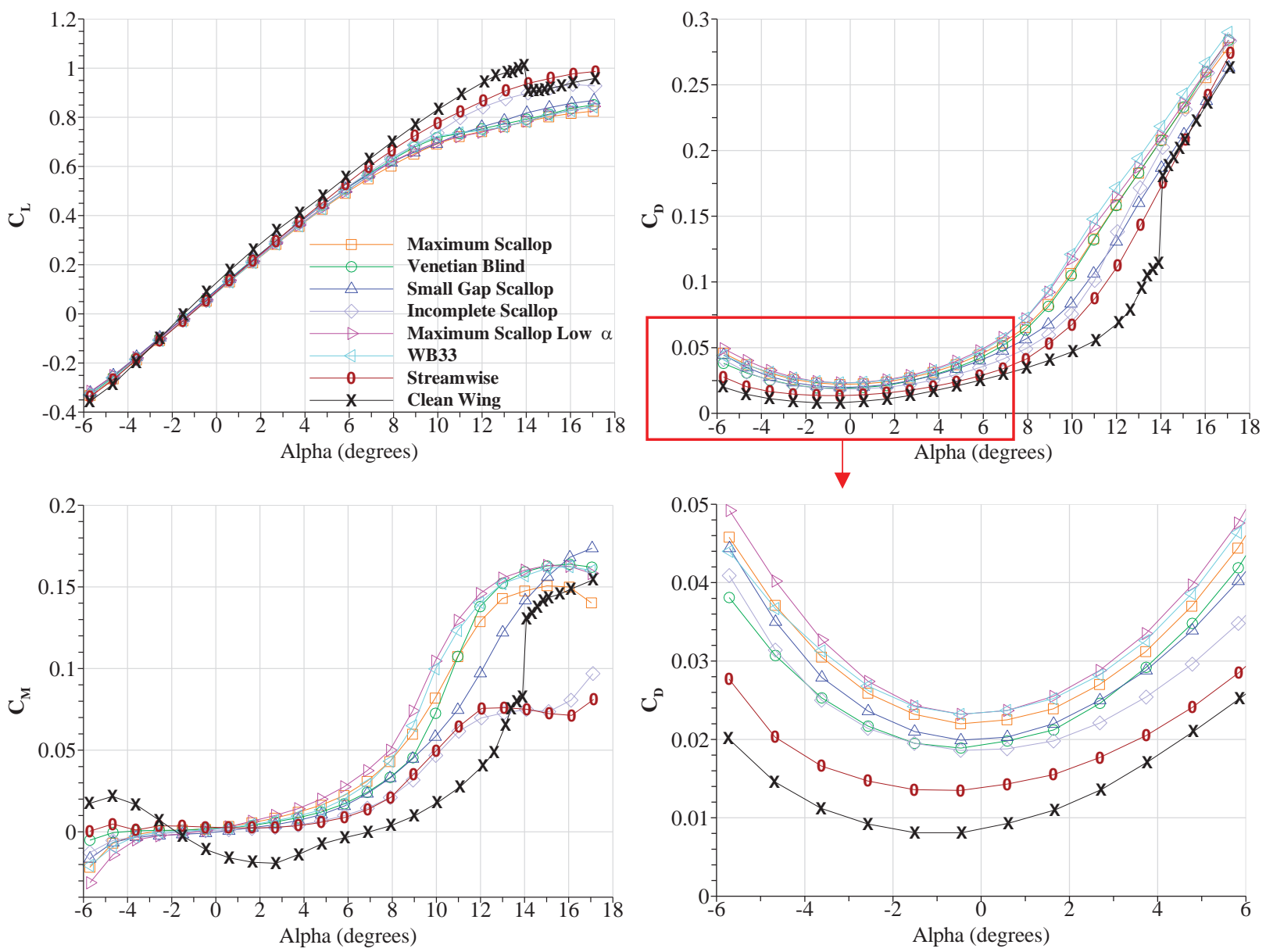

Figure 7: Comparison of aerodynamic performance data for high-fidelity configurations at $R e=$ $1.6 \times 10^{6}$ and $M=0.18$.

A summary of the aerodynamic data for each high-fidelity ice shape configuration compared to its low-fidelity counterparts and the clean wing geometry is shown in Figure 8 - Figure 12. The effects of geometric fidelity were similar for all the geometries except for the Streamwise configurations. Generally, the high-fidelity ice shapes resulted in the lowest lift at the higher angles of attack and the most drag at the lower angles of attack. The drag data observed at the lower angles of attack for the high-fidelity and 2D smooth + grit Streamwise configurations, however, were extremely similar. For the Maximum Scallop, Venetian Blind, and Maximum Scallop Low $\alpha$ geometries, adding grit to simulate ice shape roughness had a minimal effect on the lift between the 2D smooth and 2D smooth + grit configurations, but an increase in drag was observed from the 2D smooth to the 2D smooth + grit configurations for these geometries. Both the Streamwise and Maximum Scallop ice shapes were selected for more detailed study and analysis, including fluorescent oil-flow visualization and wake survey tests. The Streamwise geometry was chosen 
for its differences from the scalloped horn ice shapes while the Maximum Scallop ice shape geometry was chosen because it a large variation in geometry between the low- and high-fidelity ice shapes.
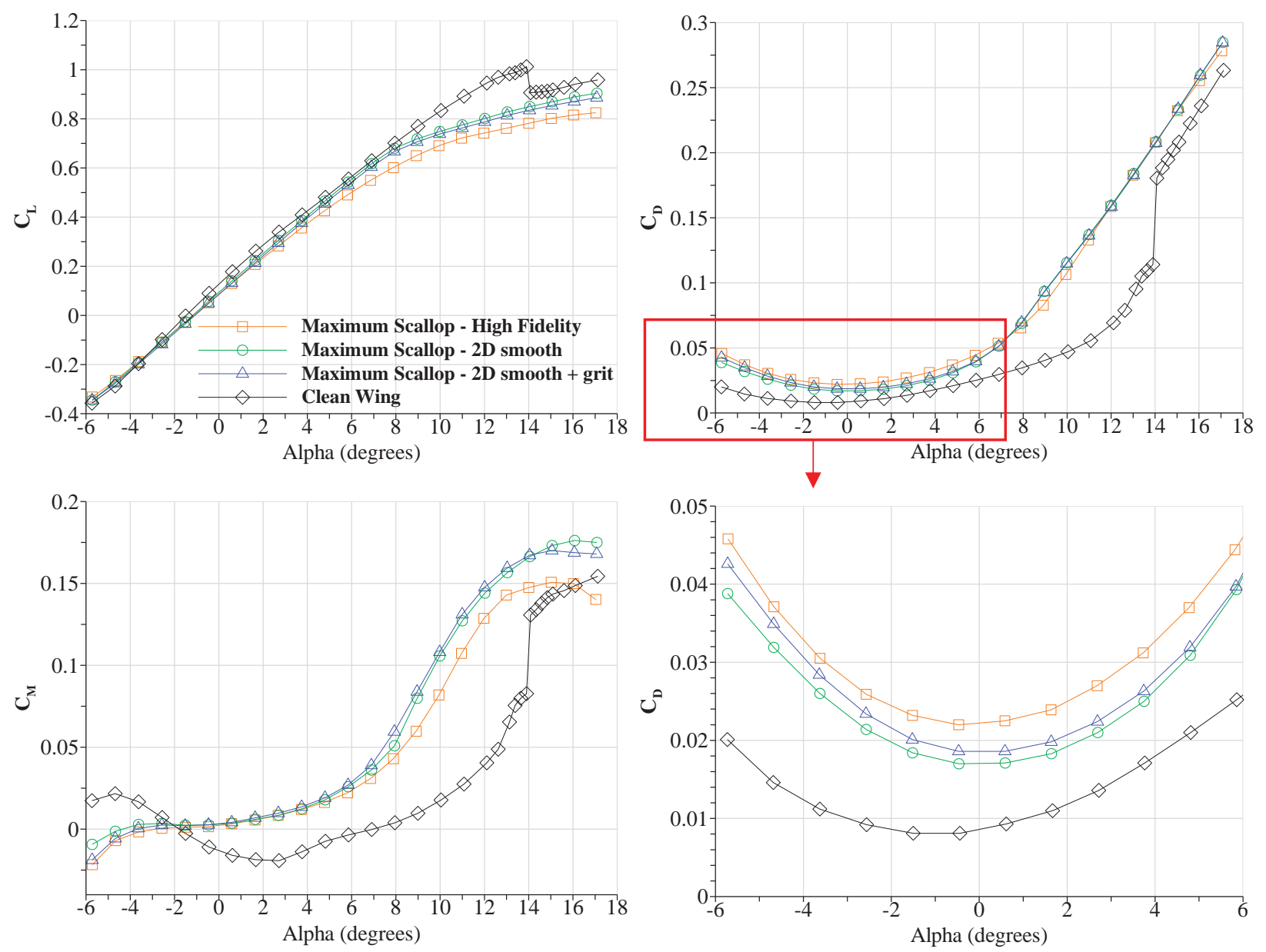

Figure 8: Force balance data for the 2D smooth, 2D smooth + grit, high-fidelity Maximum Scallop, and clean wing configurations for $R e=1.6 \times 10^{6}$ and $M=0.18$. 

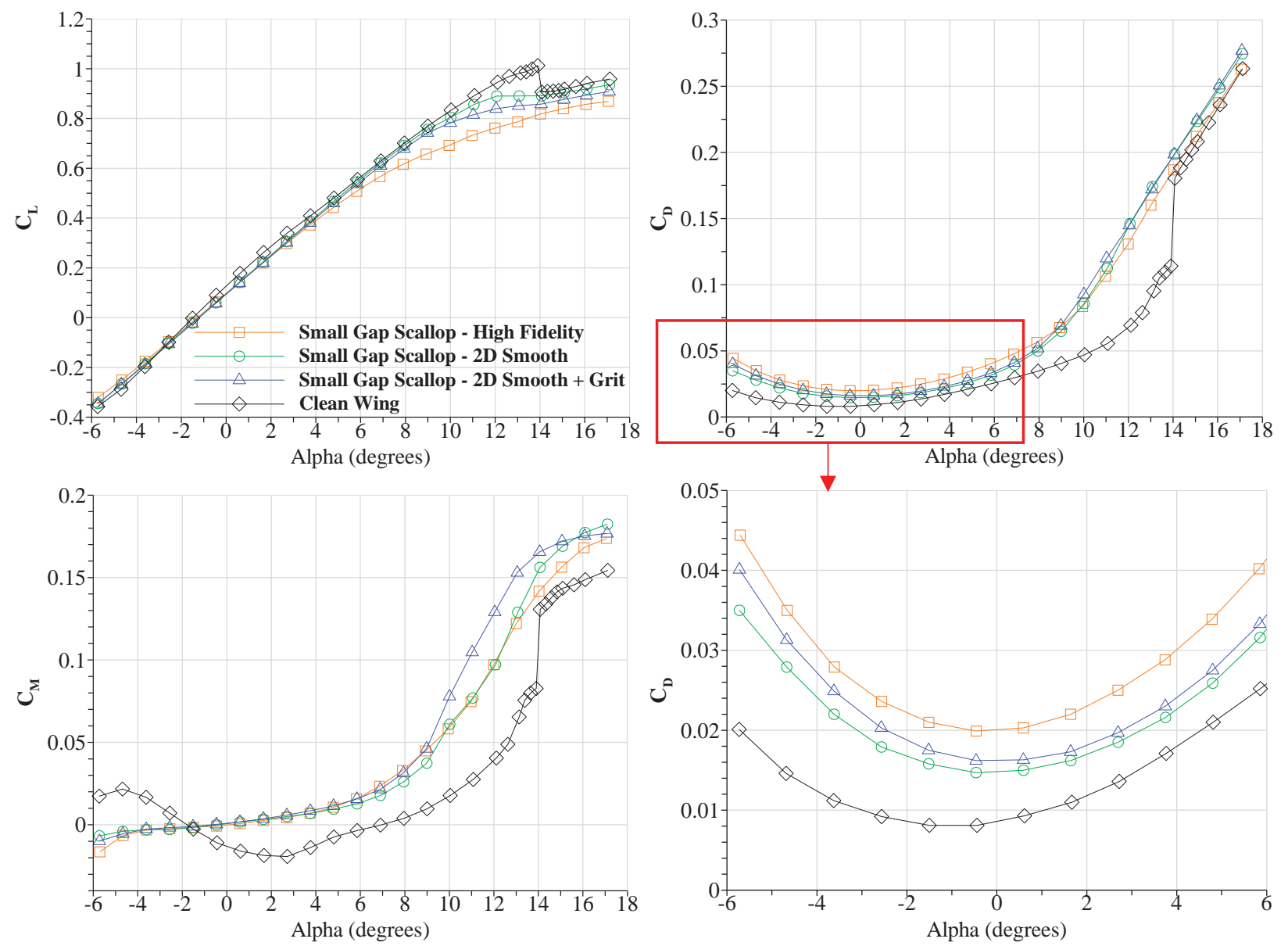

Figure 9: Force balance data for the 2D smooth, 2D smooth + grit, high-fidelity Small Gap Scallop, and clean wing configurations for $R e=1.6 \times 10^{6}$ and $M=0.18$. 

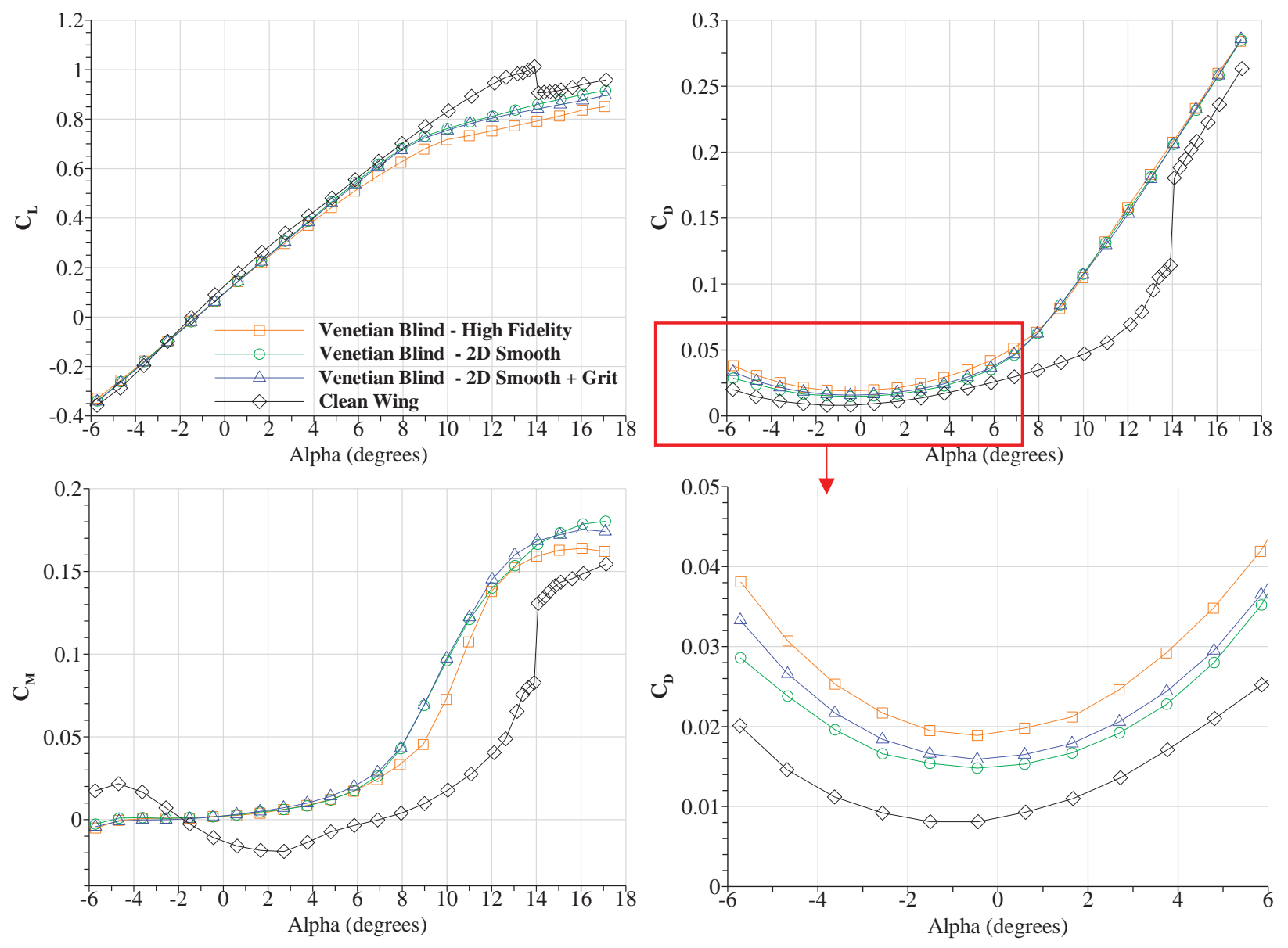

Figure 10: Force balance data for the 2D smooth, $2 D$ smooth + grit, high-fidelity Venetian Blind, and clean wing configurations for $R e=1.6 \times 10^{6}$ and $M=0.18$. 

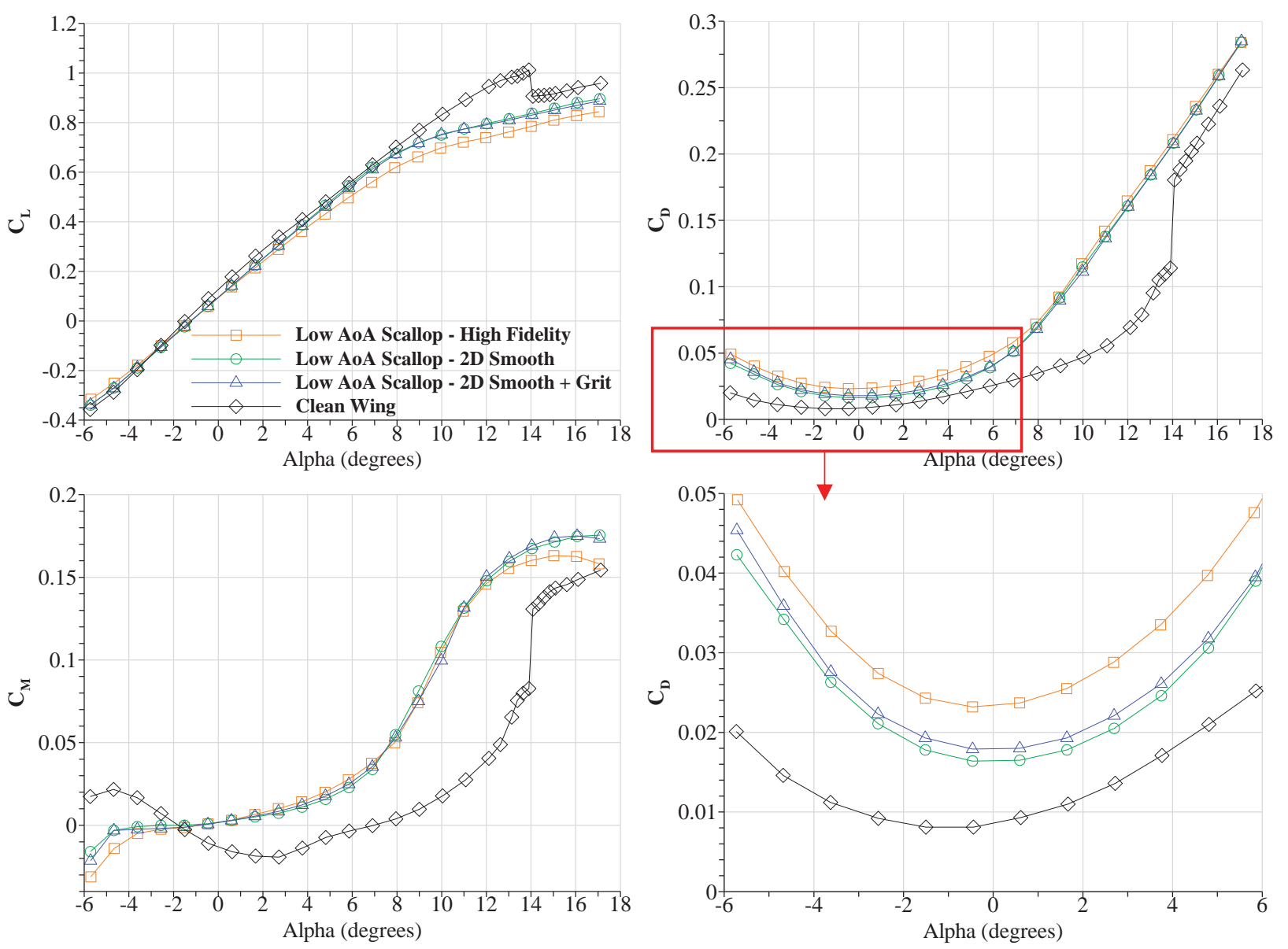

Figure 11: Force balance data for the 2D smooth, 2D smooth + grit, high-fidelity Low $\alpha$ Scallop, and clean wing configurations for $\operatorname{Re}=1.6 \times 10^{6}$ and $M=0.18$. 

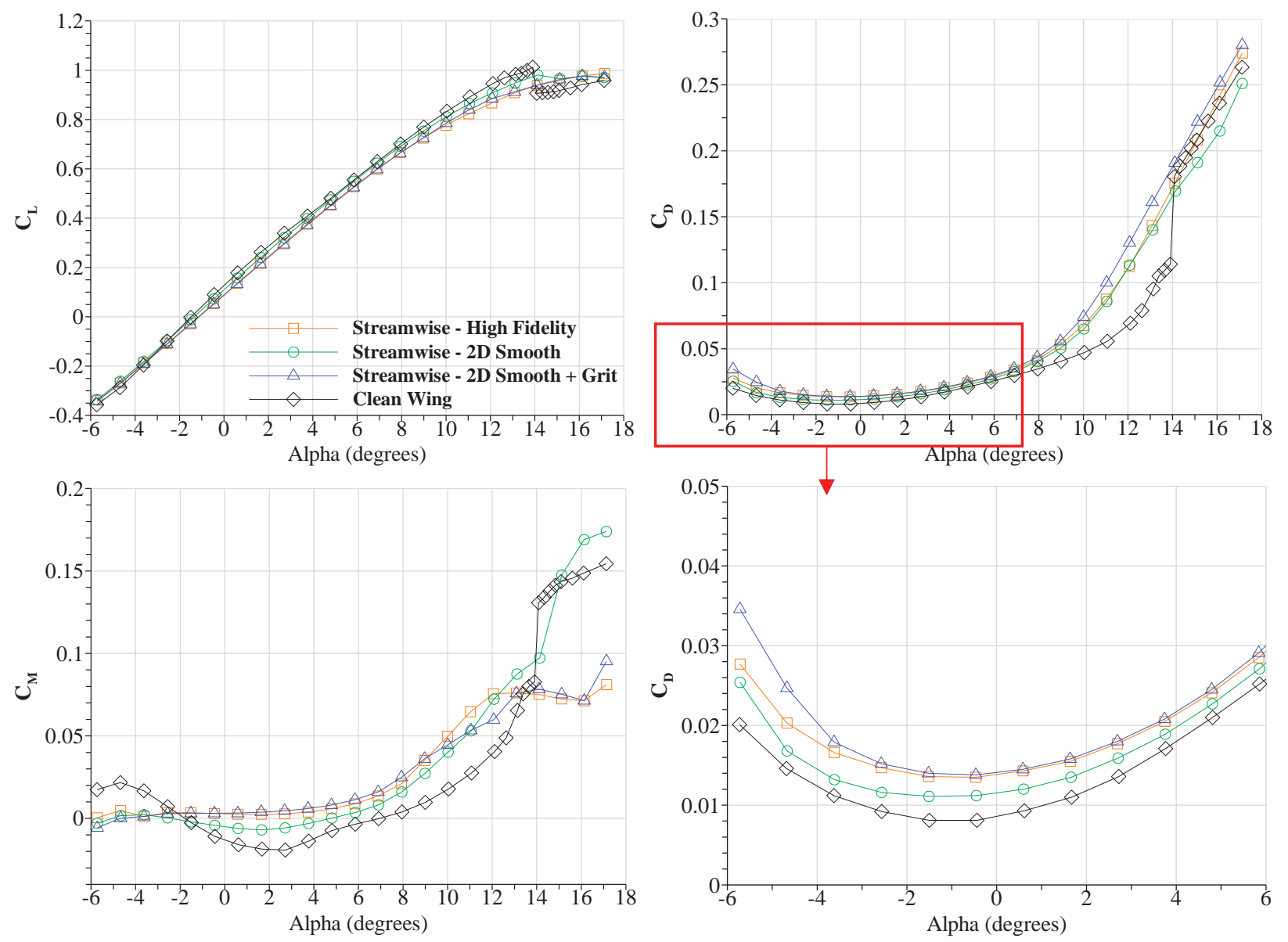

Figure 12: Force balance data for the 2D smooth, 2D smooth + grit, high-fidelity Streamwise, and clean wing configurations for $\operatorname{Re}=1.6 \times 10^{6}$ and $M=0.18$.

Photographs of the actual ice shapes and the MCCS of the Midspan hybrid model of the Streamwise and the Maximum Scallop ice accretion are shown in Figure 13. 

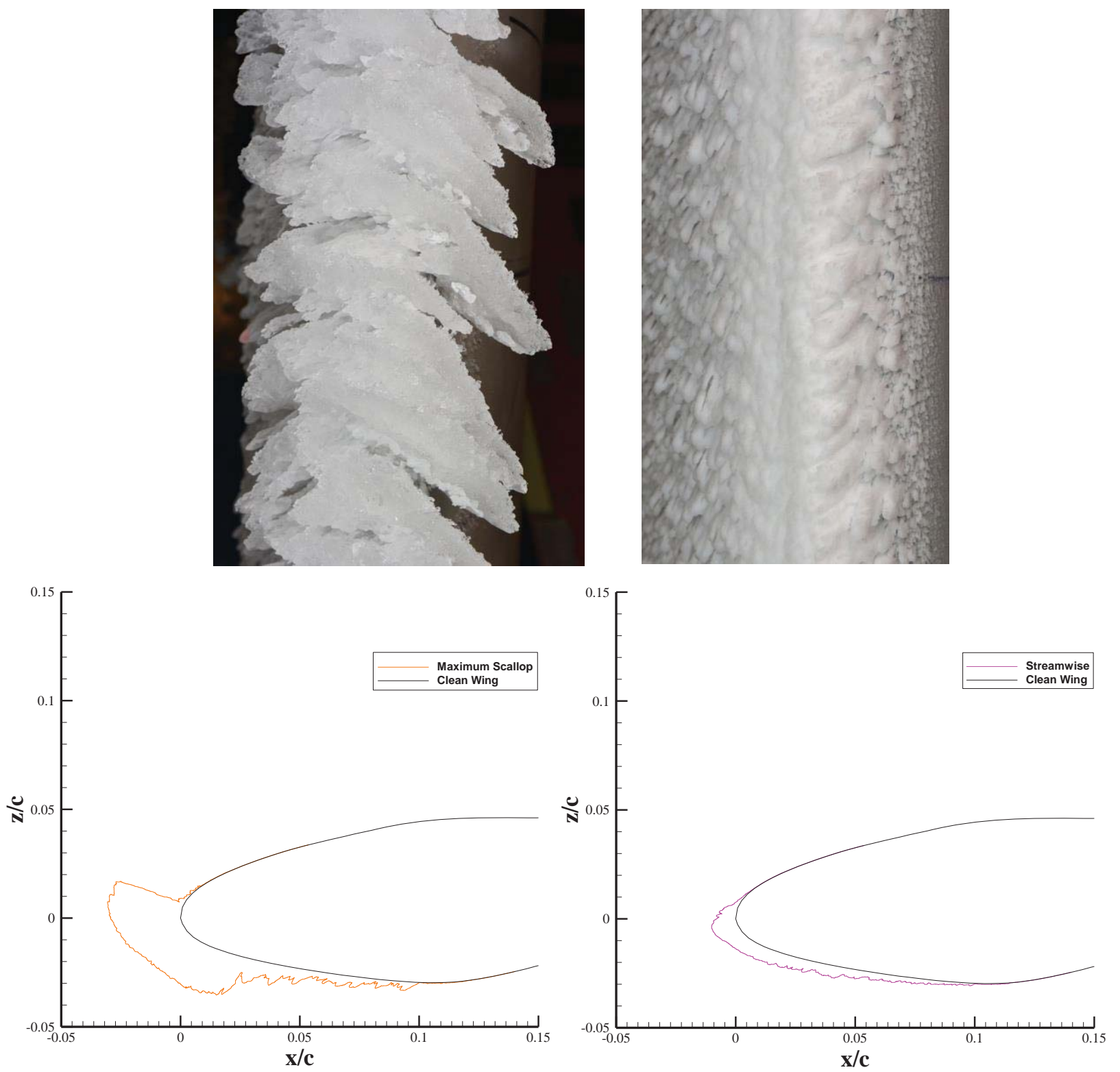

Figure 13: Maximum Scallop and Streamwise experimental ice accretion including the MCCS from the Midspan location of the CRM65.

\section{Streamwise ice shape fidelity comparison}

The oil-flow visualization, fluorescent mini-tuft visualization, and pressure contour plots for the high-fidelity Streamwise ice shape at $\alpha=6.9^{\circ}, 9.0^{\circ}$, and $12.1^{\circ}$ are shown in Figure 14 - Figure 16. Wake survey data for high-fidelity Streamwise ice shape at $\alpha=9.0^{\circ}$ is shown in Figure 17 using the test section coordinate system with the $\mathrm{y}=0$ location as the center line of the test section. The color plot represents the ratio of the axial flow velocity $(\mathrm{U})$ and the streamwise flow velocity $\left(\mathrm{U}_{\infty}\right)$, and the vectors show the velocity components. 


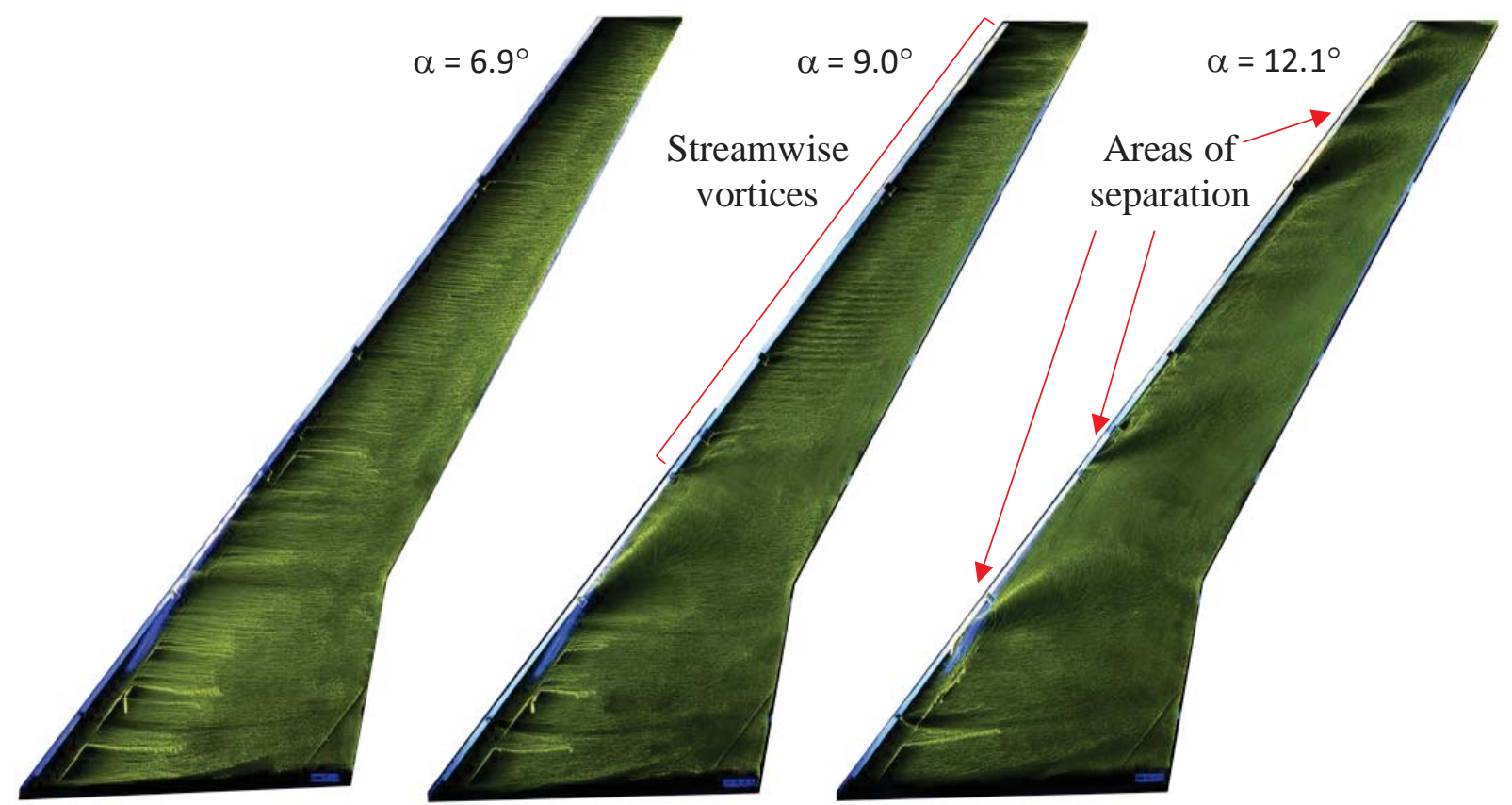

Figure 14: High-fidelity Streamwise configuration oil-flow visualization at $\operatorname{Re}=1.6 \times 10^{6}$ and $M=0.18$ for $\alpha=6.9^{\circ}, 9.0^{\circ}$, and $12.1^{\circ}$, respectively.

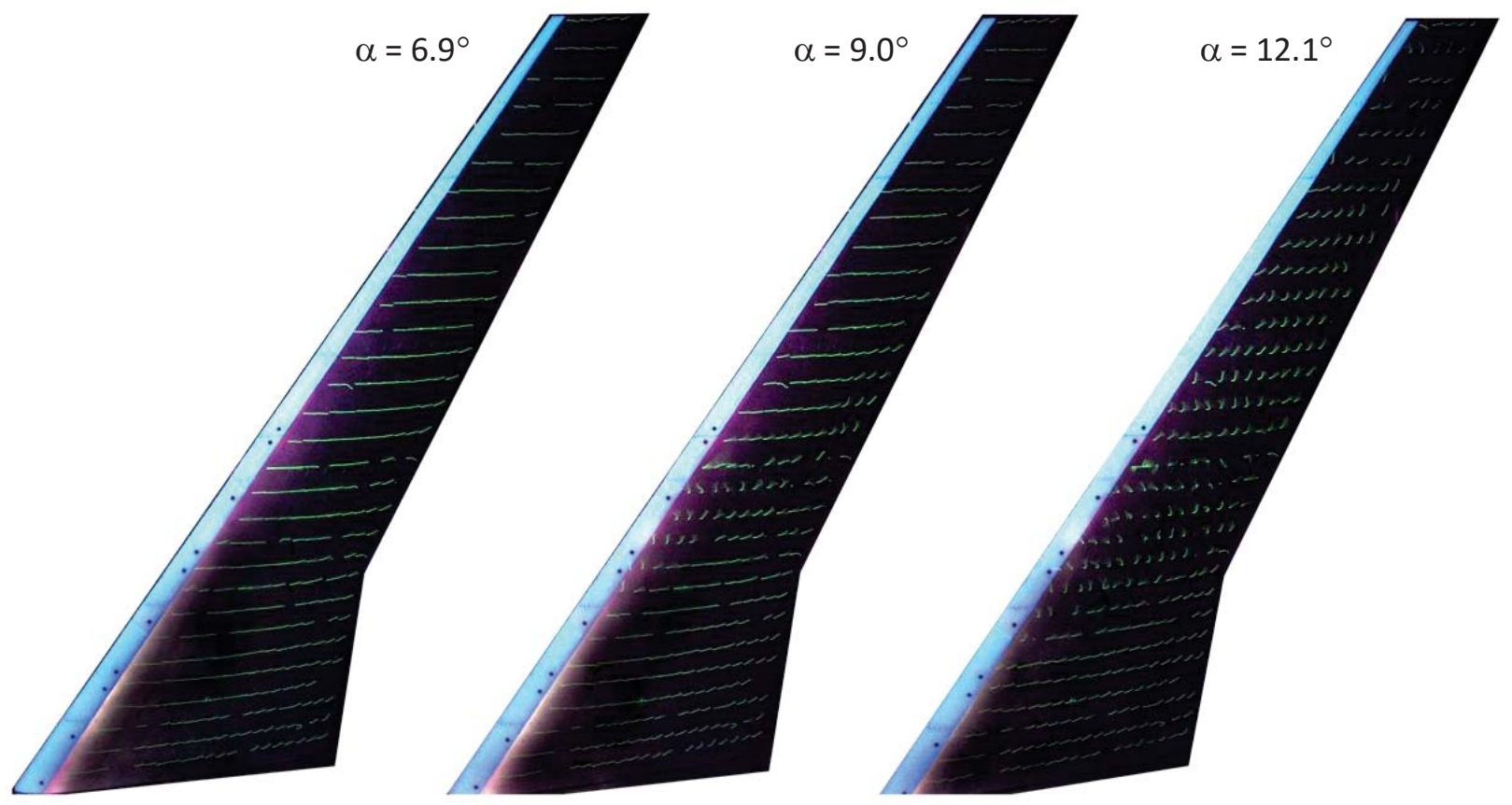

Figure 15: High-fidelity Streamwise configuration fluorescent mini-tuft visualization at $\operatorname{Re}=1.6$ $\times 10^{6}$ and $M=0.18$ for $\alpha=6.9^{\circ}, 9.0^{\circ}$, and $12.1^{\circ}$, respectively. ${ }^{17}$ 

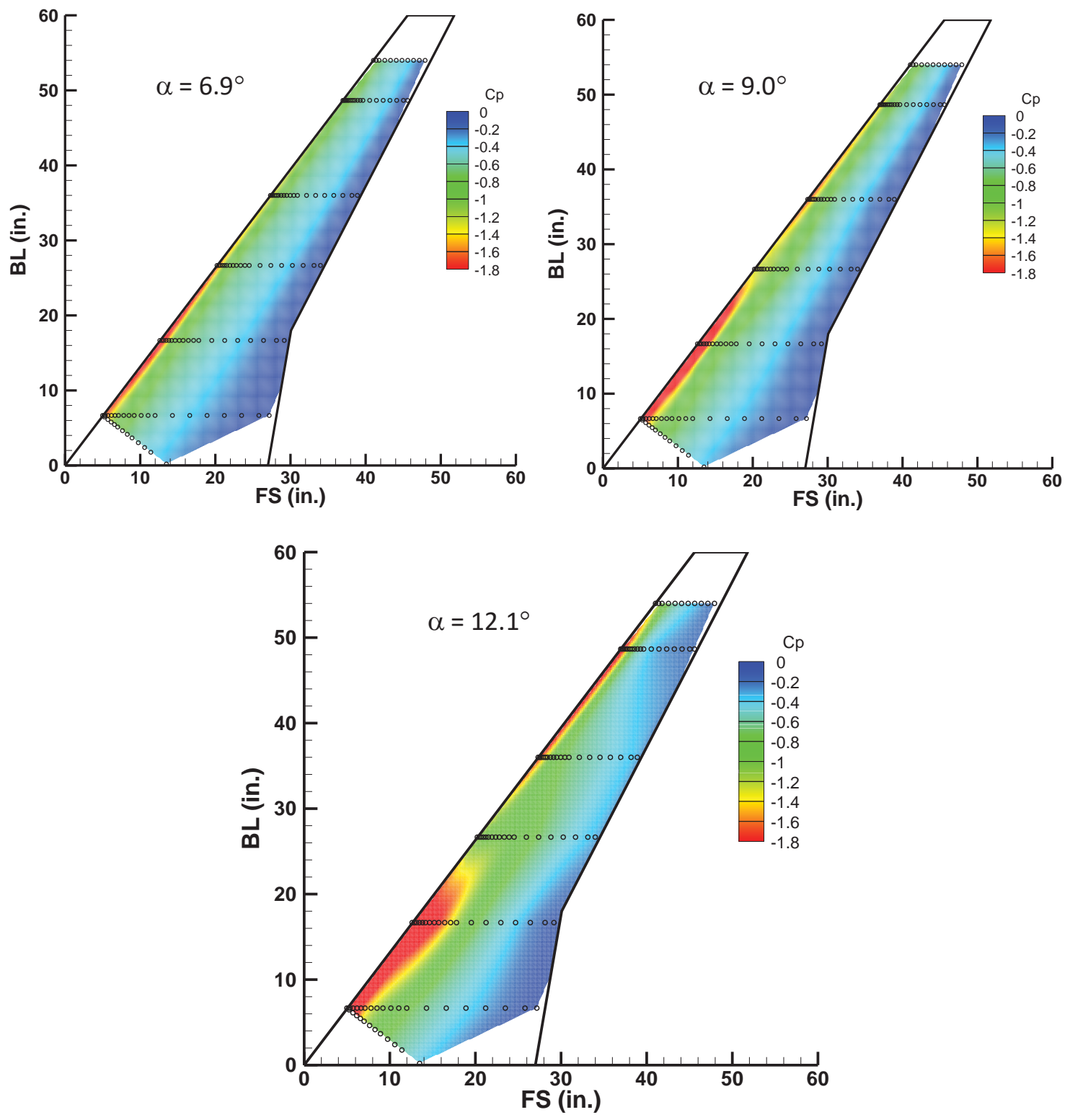

Figure 16: High-fidelity Streamwise configuration pressure contour plots at Re $=1.6 \times 10^{6}$ and $M=0.18$ for $\alpha=6.9^{\circ}, 9.0^{\circ}$, and $12.1^{\circ .17}$ 


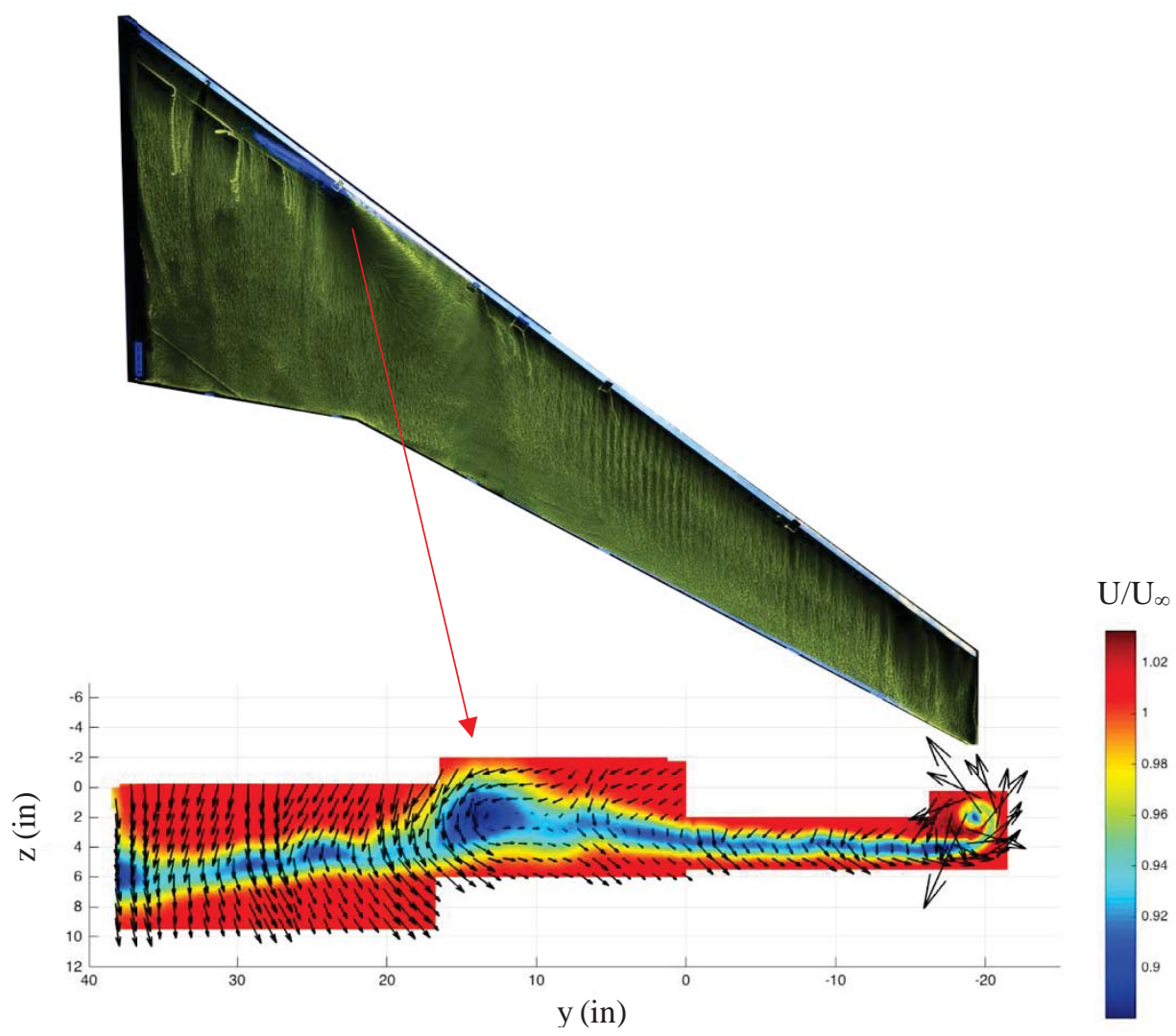

Figure 17: Wake survey velocity plot for high-fidelity Streamwise configuration at Re $=1.6 \times 10^{6}$ and $M=0.18$ for $\alpha=9.0^{\circ}$.

At $\alpha=6.9^{\circ}$ and $9.0^{\circ}$ the flow over the surface of the wing was characterized by a series of streamwise vortices in Figure 14. Some separation occurred at $\alpha=9.0^{\circ}$ near the $30 \%$ span location shown by spanwise oriented tufts in Figure 15 and in the wake as seen in Figure 17 between the 10 in and 15 in z location. A more detailed analysis of the wake for this low-Reynolds number model is shown in Lum et. al. ${ }^{24}$ A leading-edge vortex was not visible in any of the visualization techniques but a high leading-edge suction was still present in Figure 16. As the angle of attack was increased to $12.1^{\circ}$ the streamwise vortices were replaced by areas of separation and spanwise flow observed in Figure 15. The oil-flow visualization, fluorescent mini-tuft visualization, and pressure contour plots for the $2 \mathrm{D}$ smooth Streamwise ice shape at $\alpha=6.9^{\circ}, 9.0^{\circ}$, and $12.1^{\circ}$ are shown in Figure 18 - Figure 20. 


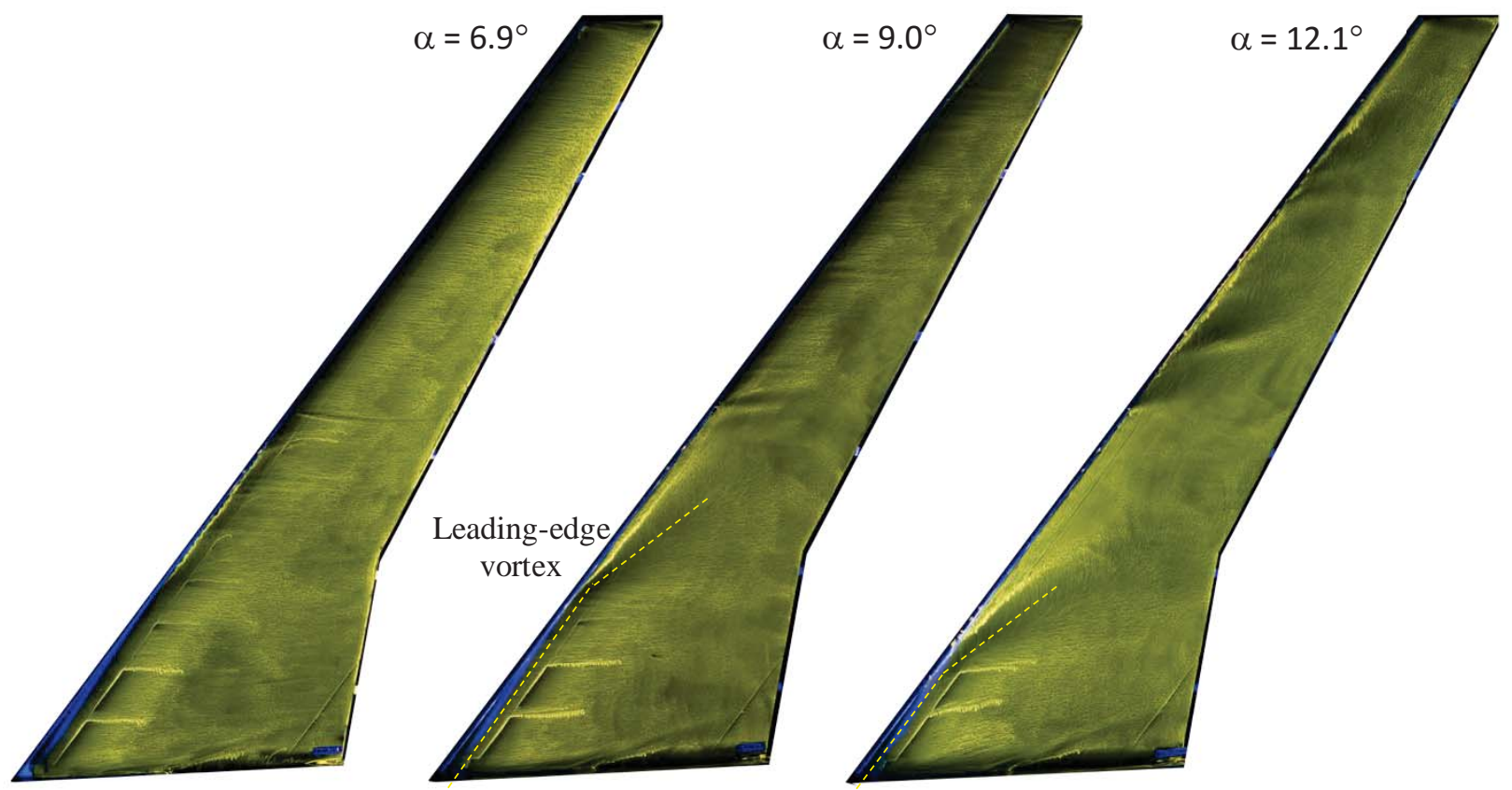

Figure 18: $2 \mathrm{D}$ smooth Streamwise configuration oil-flow visualization at $\operatorname{Re}=1.6 \times 10^{6}$ and $\mathrm{M}$ $=0.18$ for $\alpha=6.9^{\circ}, 9.0^{\circ}$, and $12.1^{\circ}$, respectively.

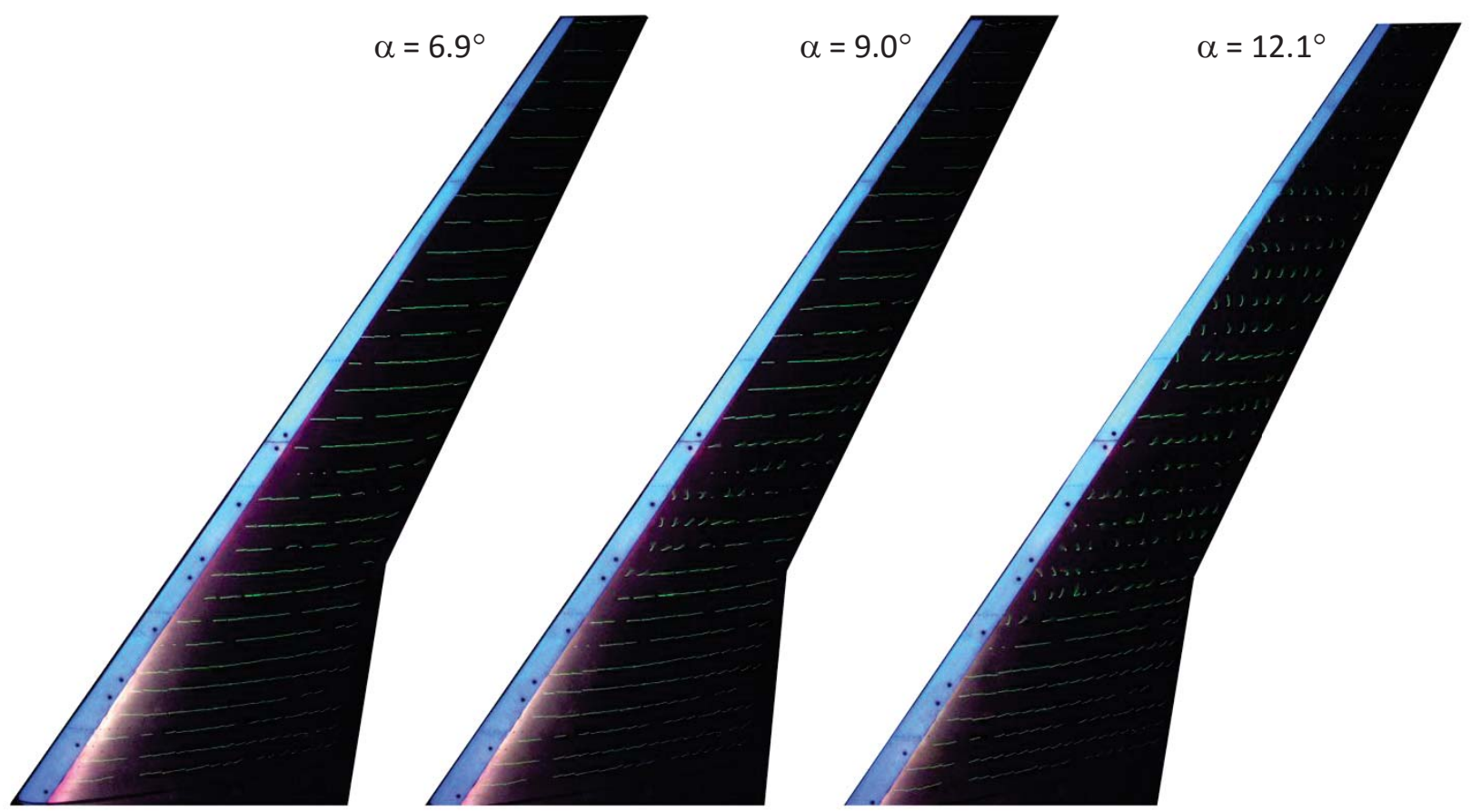

Figure 19: 2D smooth Streamwise configuration fluorescent mini-tuft visualization at $R e=1.6 \times$ $10^{6}$ and $M=0.18$ for for $\alpha=6.9^{\circ}, 9^{\circ} 0^{\circ}$, and $12.1^{\circ}$, respectively. ${ }^{17}$ 

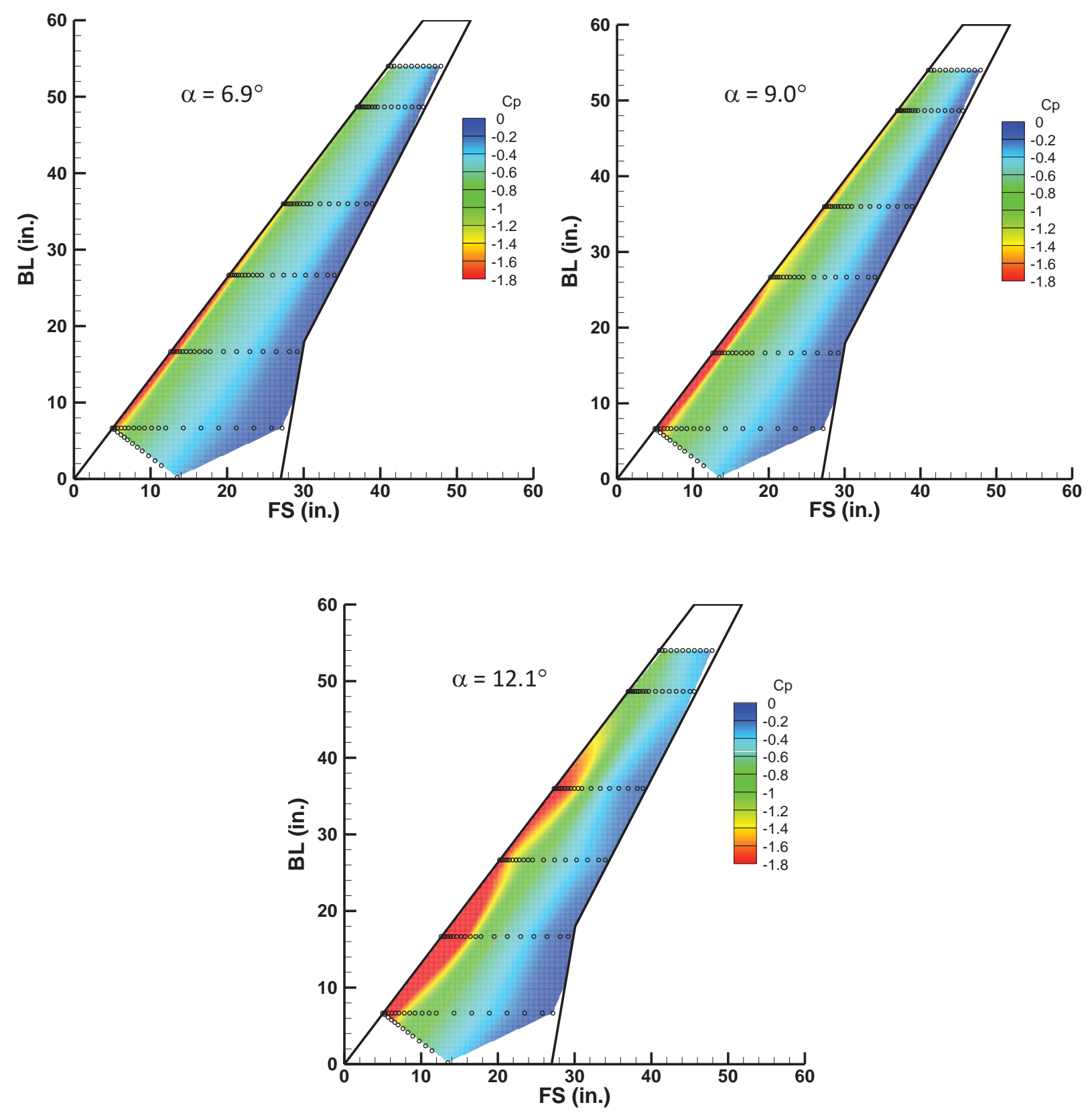

Figure 20: $2 D$ smooth Streamwise configuration pressure contour plots at $\operatorname{Re}=1.6 \times 10^{6}$ and $M=0.18$ for $\alpha=6.9^{\circ}, 9.0^{\circ}$, and $12.1 .^{17}$

The oil-flow visualization of the 2D smooth Streamwise configuration did not show the streamwise vortices that were present in the high-fidelity Streamwise configuration in Figure 14, but features of a leading-edge vortex were present. The fluorescent mini-tuft data in Figure 15 and Figure 19 also showed many similarities between the two configurations. At $\alpha=9.0^{\circ}$, an area of separation for both the 2D smooth and high-fidelity Streamwise configurations was present at 
approximately the 30\% span location as seen in Figure 19. While the pressure contour plots between the 2D smooth and high-fidelity configurations agreed at the lower angles of attack, a higher suction near the leading edge of the 2D smooth configuration at $\alpha=12.1^{\circ}$ may have led to the differences in the force balance data. These differences were observed as a higher lift and lower moment for the 2D smooth configuration than the high-fidelity configuration in Figure 11. Fluorescent mini-tuft visualization and pressure contour plots for the 2D smooth + grit Streamwise ice shape at $\alpha=6.9^{\circ}, 9.0^{\circ}$, and $12.1^{\circ}$ are shown in Figure 21 and Figure 22. Wake survey data for the 2D smooth + grit Streamwise ice shape at $\alpha=9.0^{\circ}$ is shown in Figure 23. Oil-flow visualization was not conducted at these angles of attack for this case.

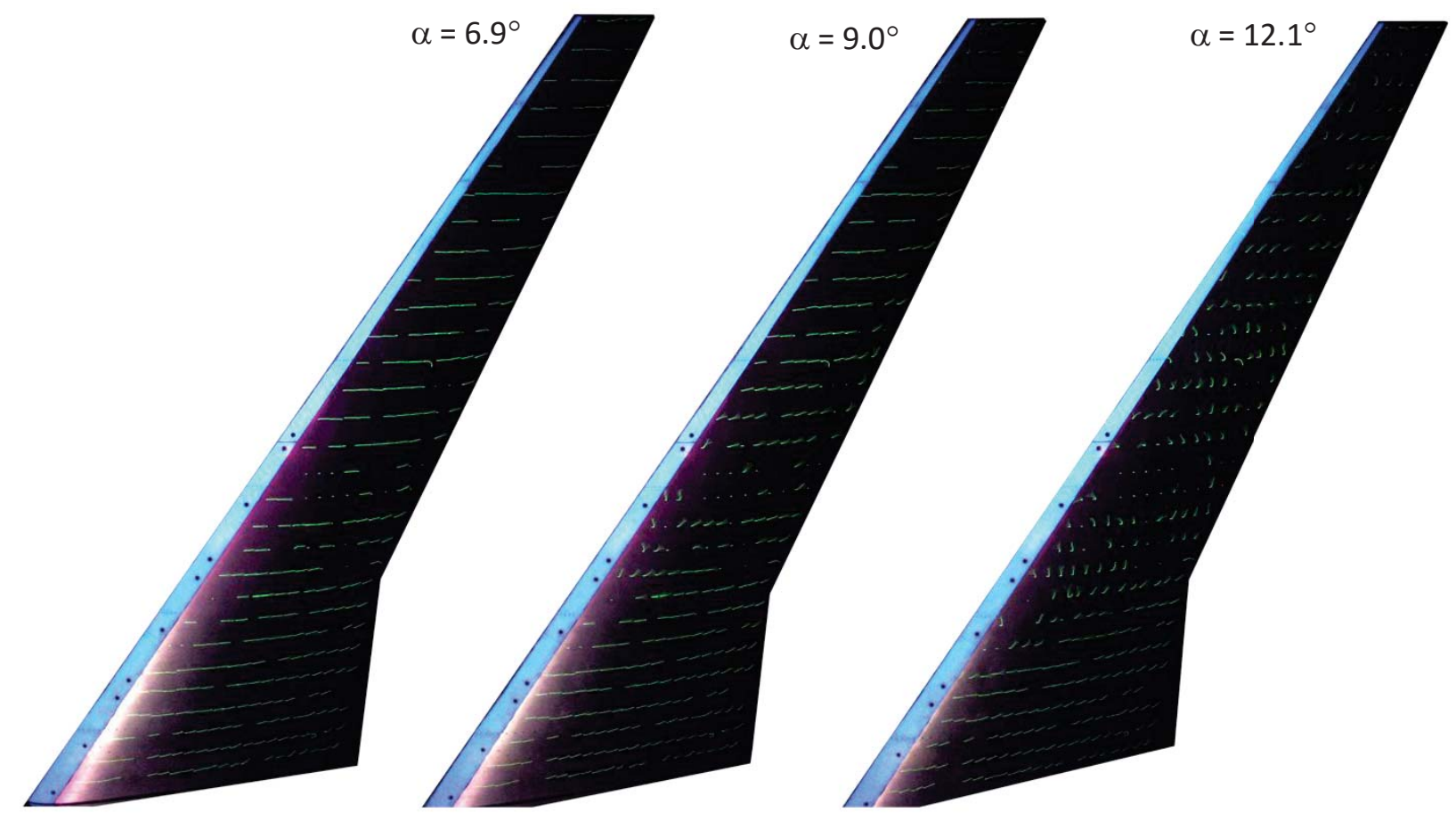

Figure 21: $2 D$ smooth + grit Streamwise configuration fluorescent mini-tuft visualization at Re $=1.6 \times 10^{6}$ and $M=0.18$ for for $\alpha=6.9^{\circ}, 9.0^{\circ}$, and $12.1^{\circ}$, respectively..$^{17}$ 

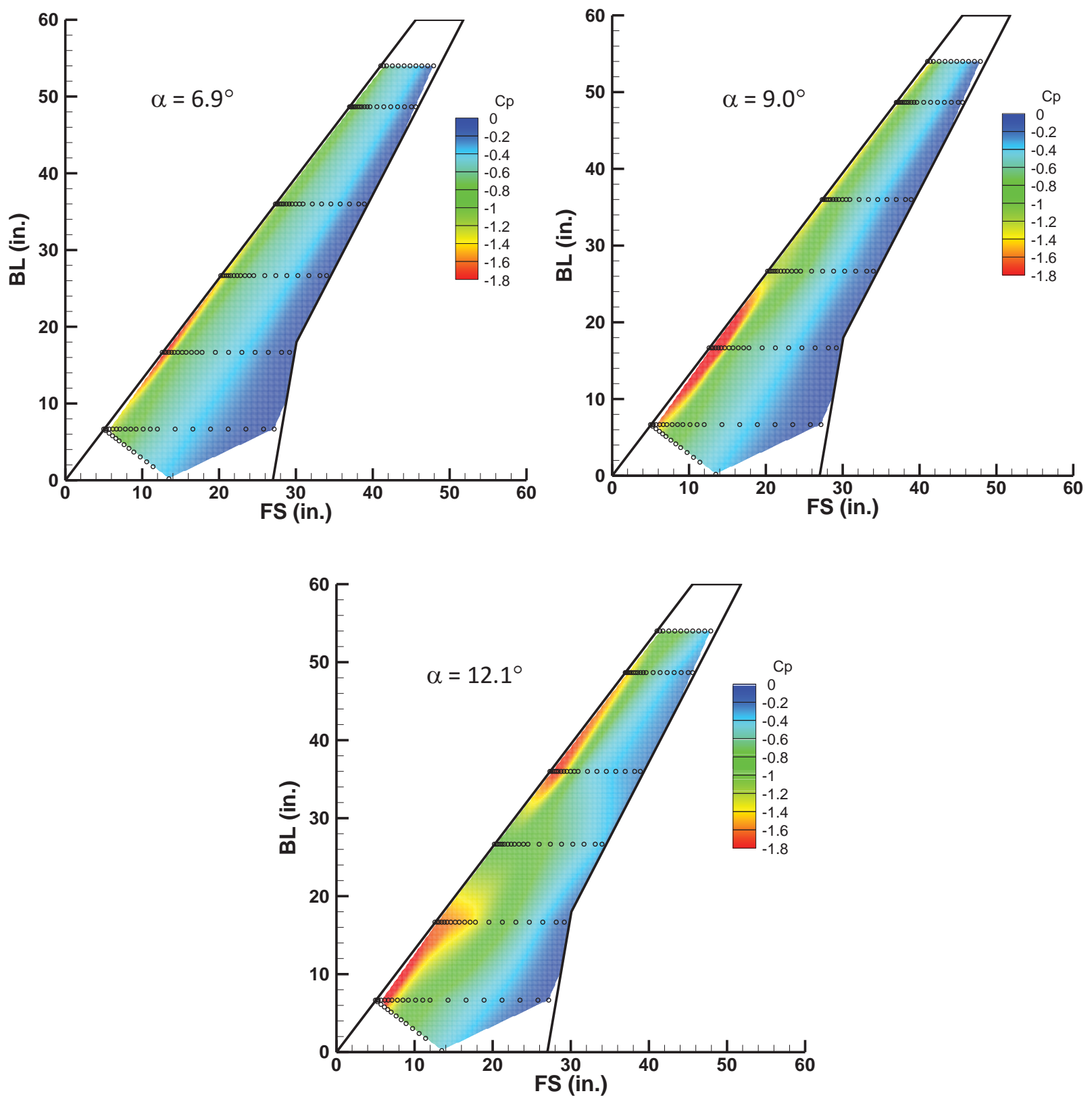

Figure 22: $2 D$ smooth + grit Streamwise configuration pressure contour plots at Re $=1.6 \times 10^{6}$ and $M=0.18$ for $\alpha=6.9^{\circ}, 9.0^{\circ}$, and $12.1 .^{17}$ 


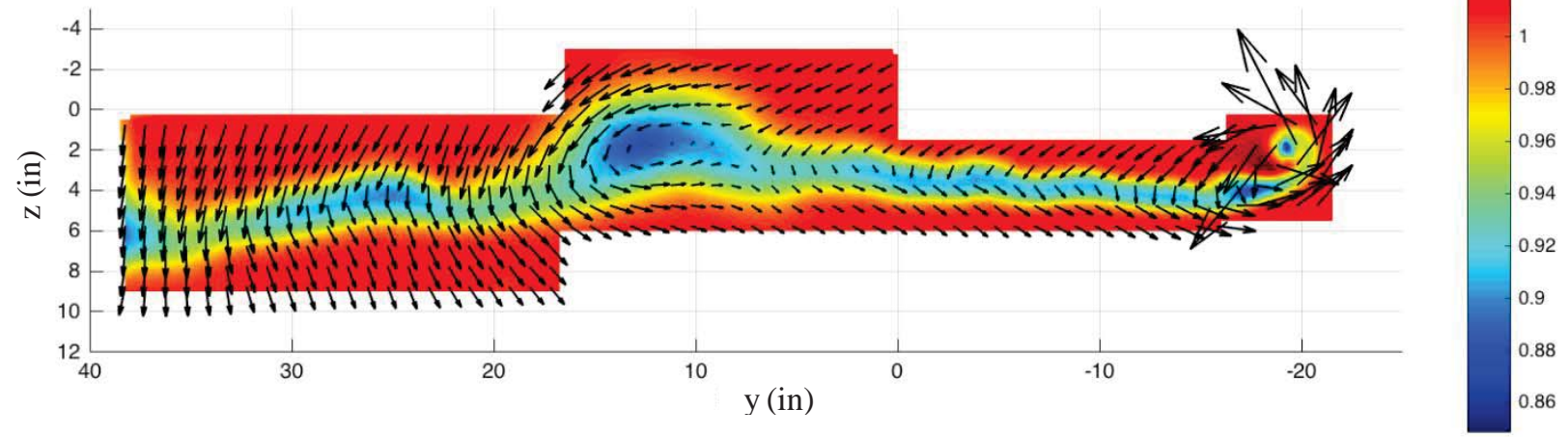

Figure 23: Wake survey velocity plot for 2D smooth + grit Streamwise configuration at $\mathrm{Re}=1.6$ $\times 10^{6}$ and $M=0.18$ for $\alpha=9.0^{\circ}$.

The fluorescent mini-tuft visualization for the 2D smooth + grit Streamwise configuration in Figure 21 was extremely similar the 2D smooth Streamwise configuration in Figure 19 and the high-fidelity Streamwise configuration in Figure 15. The pressure contour plots between the two low-fidelity configurations, however, showed some significant differences between the pressures near the leading edge. In addition, both the 2D smooth + grit and high-fidelity Streamwise configurations showed a large area of separation near the $30 \%$ span of the wing in the wake survey data as seen in Figure 17 and Figure 23. The 2D smooth + grit configuration had pressure data more similar to the high-fidelity Streamwise configuration. Both have two distinct areas of high suction near the leading edge of the wing whereas the 2D smooth configuration shows a single large area of high suction inboard of the $75 \%$ span location. These similarities in leading-edge suction and the wake between the 2D smooth + grit and high-fidelity ice shapes may have played a role in the similarities in the aerodynamic data between the two configurations. Previous 2D airfoil tests with streamwise ice shapes produced similar results and it was concluded that the aerodynamic effects for streamwise ice shapes were due to roughness characteristics of the ice shape and not the ice shape geometry itself for the 2D ice shapes tested. ${ }^{7}$

\section{Maximum Scallop ice shape fidelity comparison}

The oil-flow visualization, fluorescent mini-tuft visualization, and pressure contour plots for the high-fidelity Maximum Scallop ice shape at $\alpha=4.8^{\circ}, 7.9^{\circ}$, and $8.9^{\circ}$ are shown in Figure $24-$ Figure 26. Wake survey data for the high-fidelity Maximum Scallop ice shape at $\alpha=7.9^{\circ}$ is shown in Figure 23. 


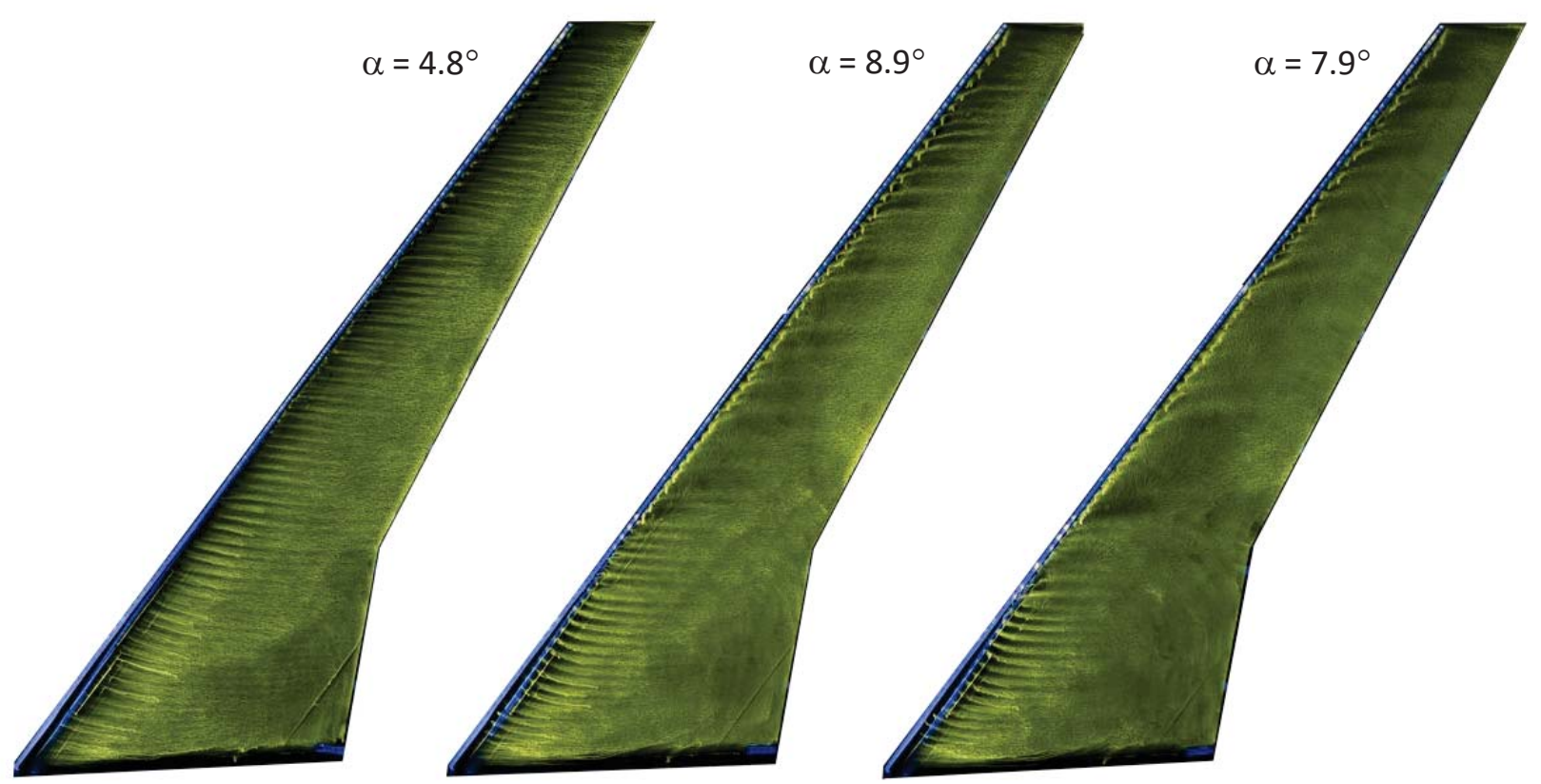

Figure 24: High -fidelity Maximum Scallop configuration oil-flow visualization at $\operatorname{Re}=1.6 \times$ $10^{6}$ and $M=0.18$ for $\alpha=4.8^{\circ}, 7.9^{\circ}$, and $8.9^{\circ}$, respectively. ${ }^{17}$

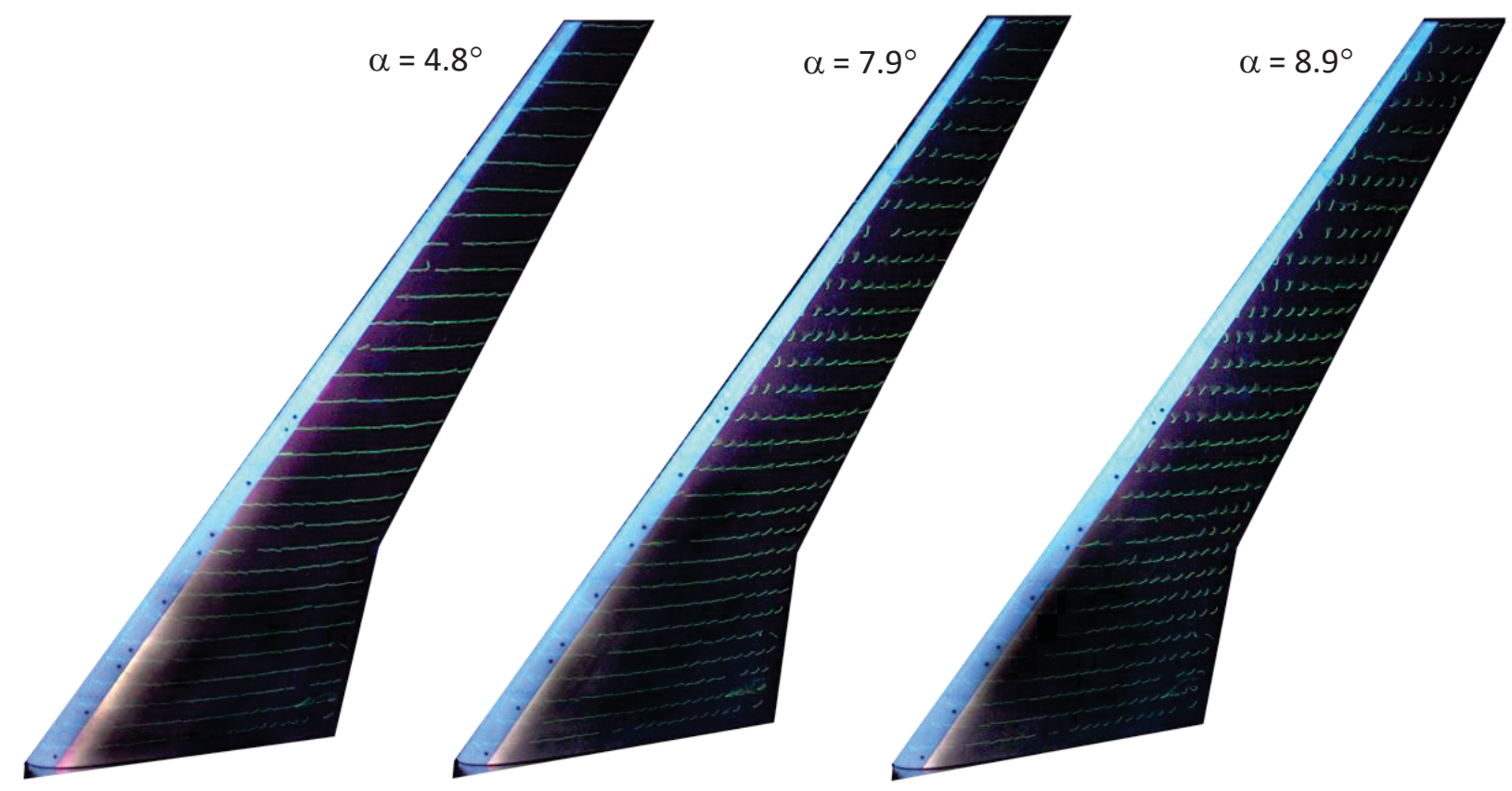

Figure 25: High-fidelity Maximum Scallop fluorescent mini-tuft visualization at $\operatorname{Re}=1.6 \times 10^{6}$ and $M=0.18$ for $\alpha=4.8^{\circ}, 7.9^{\circ}$, and $8.9^{\circ}$, respectively. ${ }^{17}$ 

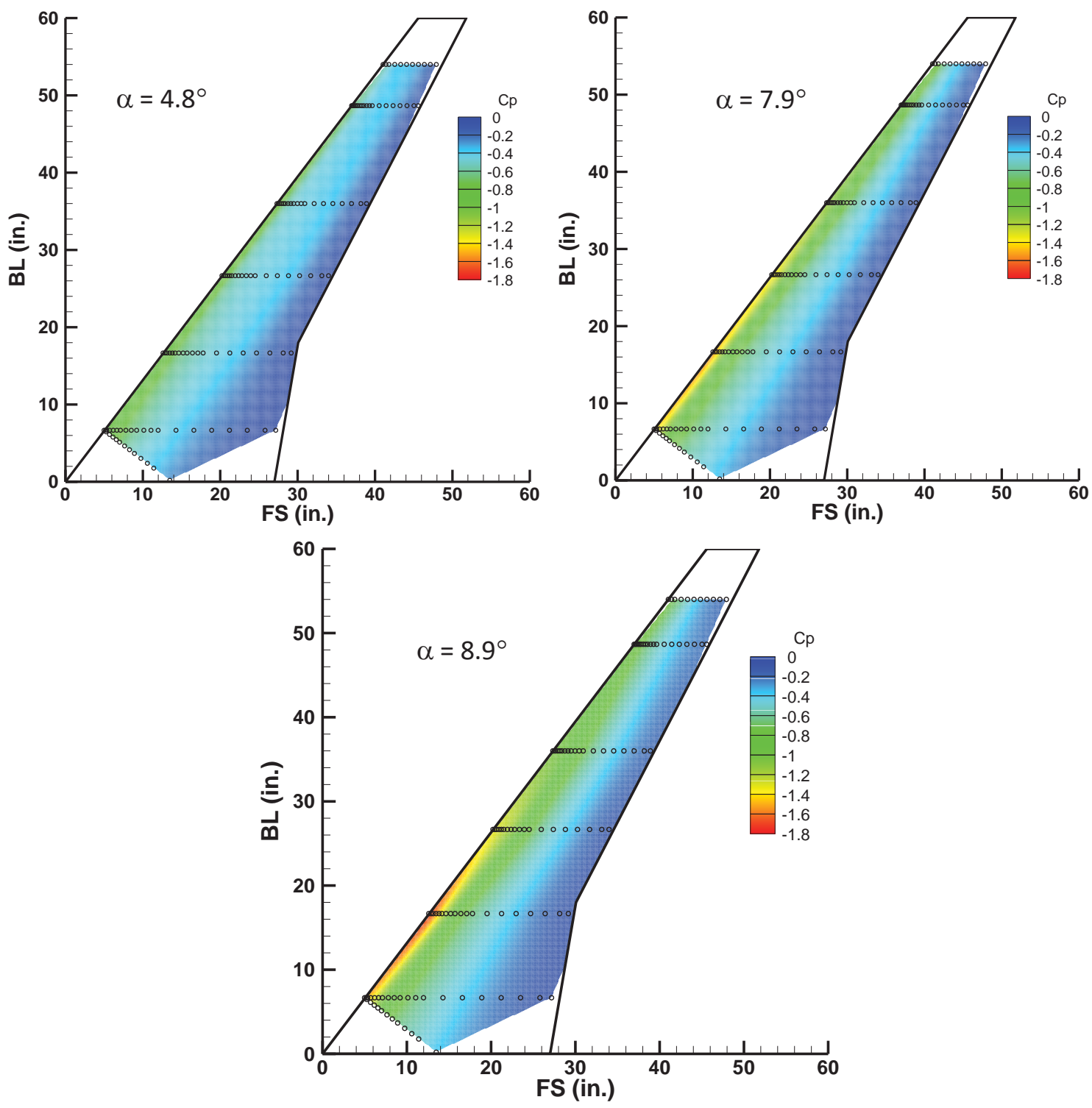

Figure 26: High-fidelity Maximum Scallop configuration pressure contour plots at Re $=1.6 \times$ $10^{6}$ and $M=0.18$ for $\alpha=4.8^{\circ}, 7.9^{\circ}$, and $8.9^{\circ} .^{17}$ 


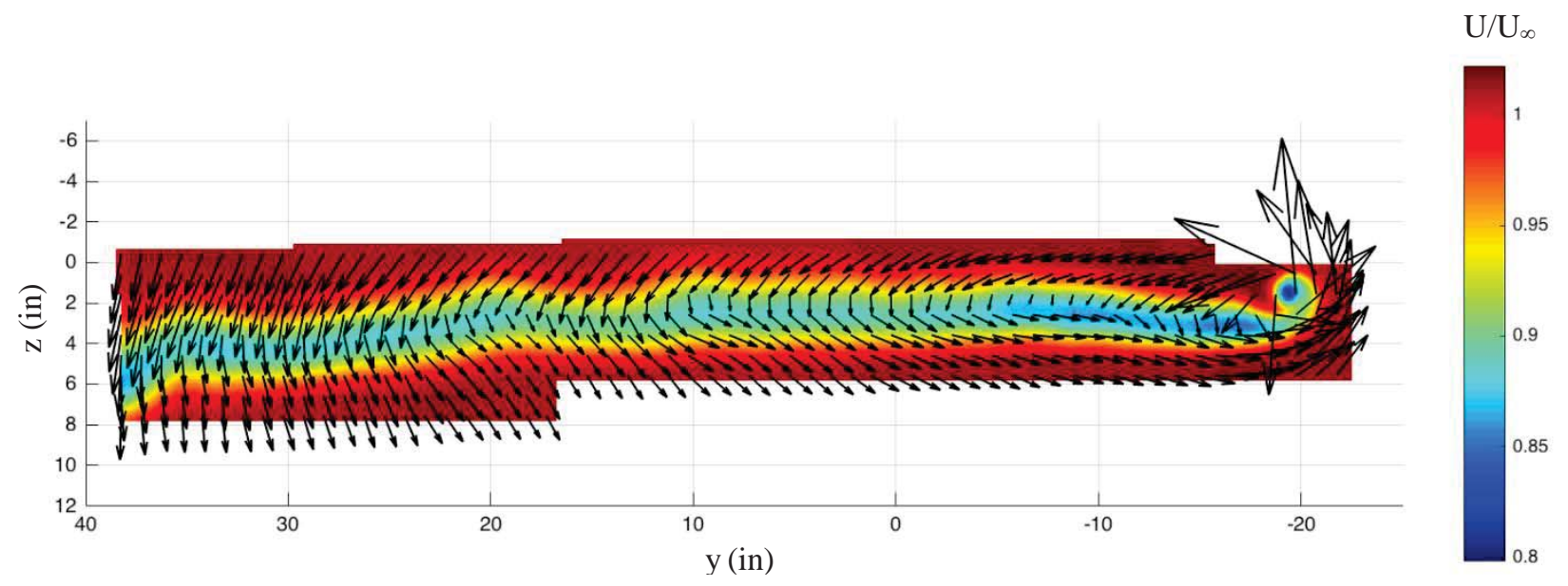

Figure 27: Wake survey velocity plot for high-fidelity Maximum Scallop configuration at Re = $1.6 \times 10^{6}$ and $M=0.18$ for $\alpha=7.9^{\circ}$.

At $\alpha=4.8^{\circ}$ the flow over the surface of the wing was characterized by a series of streamwise vortices in Figure 24. These streamwise vortices in Figure 24 were larger and more evenly spaced than the streamwise vortices on the Streamwise ice shape configuration and were most likely created by the gaps between the scallops of the Maximum Scallop geometry which acted like vortex generators. This periodicity of streamwise vortices between scallops was previously observed for a scalloped horn ice shape geometry on a swept-wing model tested in the $3 \mathrm{ft} x 4 \mathrm{ft}$ wind tunnel at UIUC ${ }^{17}$. The individual streamwise vortices were not visible in the wake survey data at $\alpha=7.9^{\circ}$ in Figure 27 which did not show any large areas of separation and was consistent with the other visualization data available for this configuration. For the high-fidelity Maximum Scallop configuration, features of a leading-edge vortex were visible between some of the streamwise vortices in the oil-flow visualization in Figure 24 and the fluorescent mini-tufts near the leading edge of the model in Figure 25 show some spanwise flow. While these observations suggest the presence of a leading-edge vortex, there is not enough evidence from these flow visualization techniques to fully describe the flowfield. As the angle of attack was increased, the streamwise vortices were replaced by areas of separation and spanwise flow observed in the tufts in Figure 25. The oil-flow visualization, fluorescent mini-tuft visualization, and pressure contour plots for the 2D smooth Maximum Scallop ice shape at $\alpha=4.8^{\circ}, 7.9^{\circ}$, and $8.9^{\circ}$ are shown in Figure 28 - Figure 30 . 


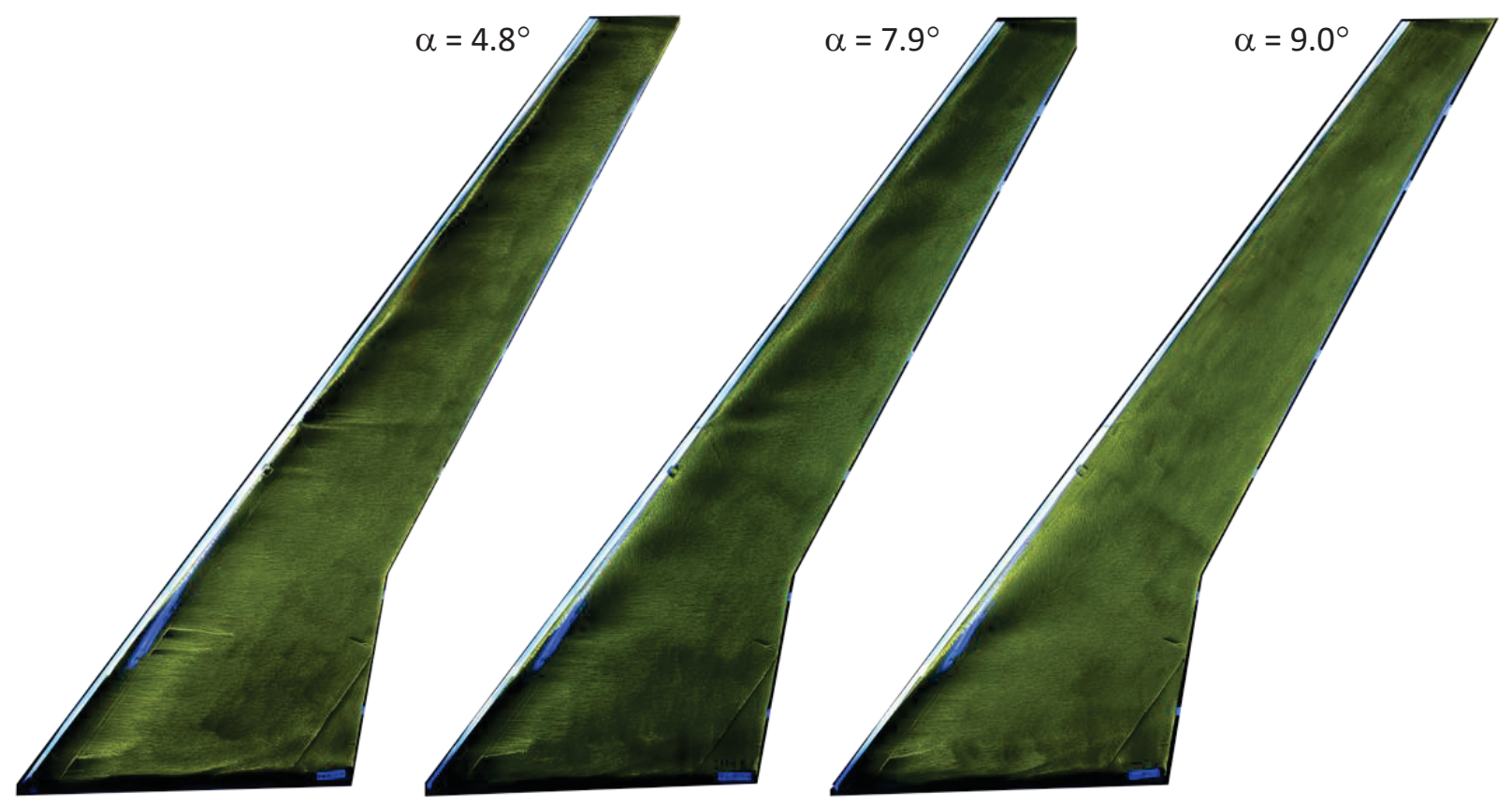

Figure 28: $2 D$ smooth Maximum Scallop configuration oil-flow visualization at Re $=1.6 \times 10^{6}$ and $M=0.18$ for $\alpha=4.8^{\circ}, 7.9^{\circ}$, and $9.0^{\circ}$, respectively. ${ }^{17}$

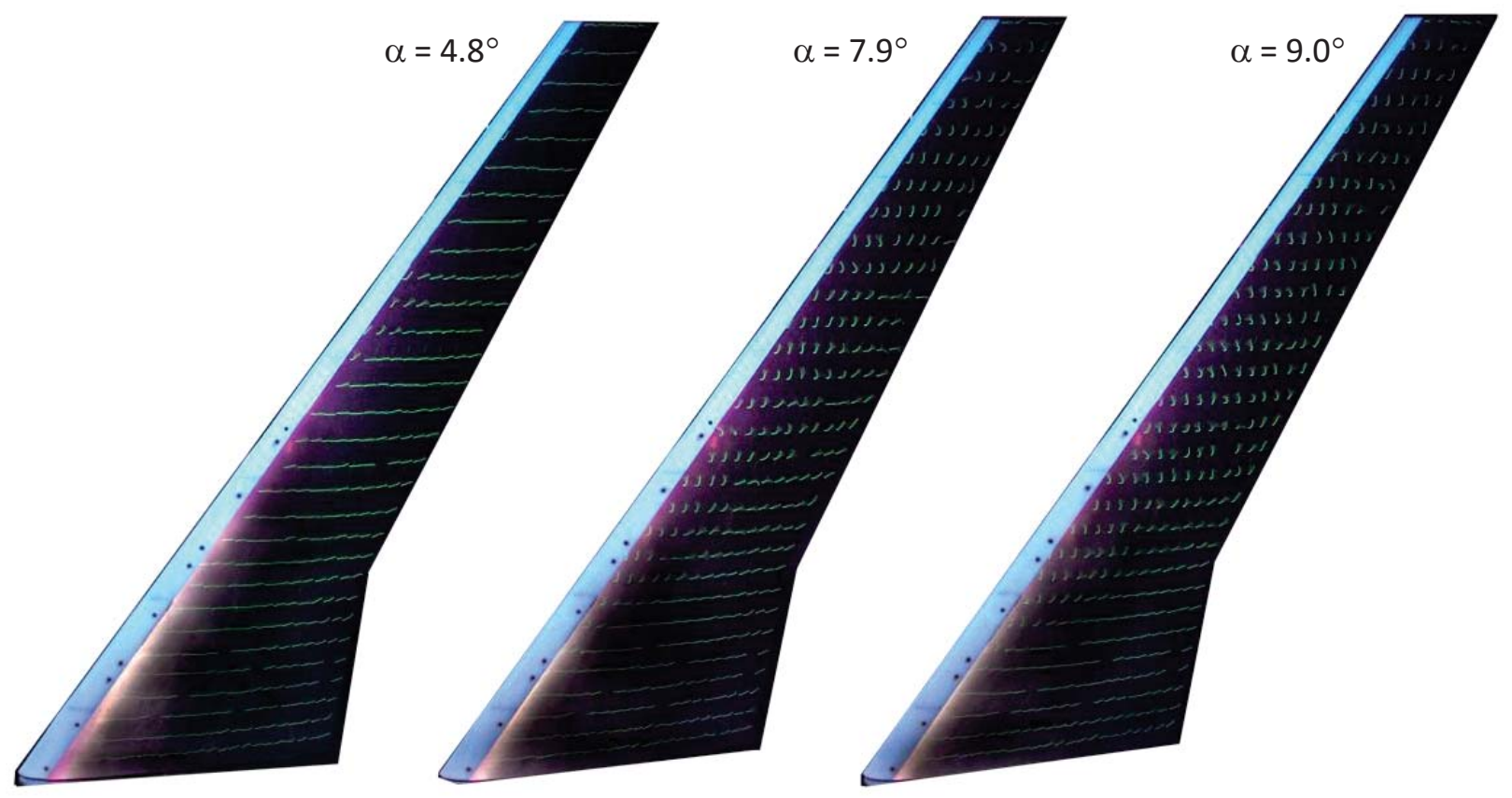

Figure 29: 2D smooth Maximum Scallop configuration fluorescent mini-tuft visualization at Re $=1.6 \times 10^{6}$ and $M=0.18$ for $\alpha=4.8^{\circ}, 7.9^{\circ}$, and $9.0^{\circ}$, respectively. ${ }^{17}$ 

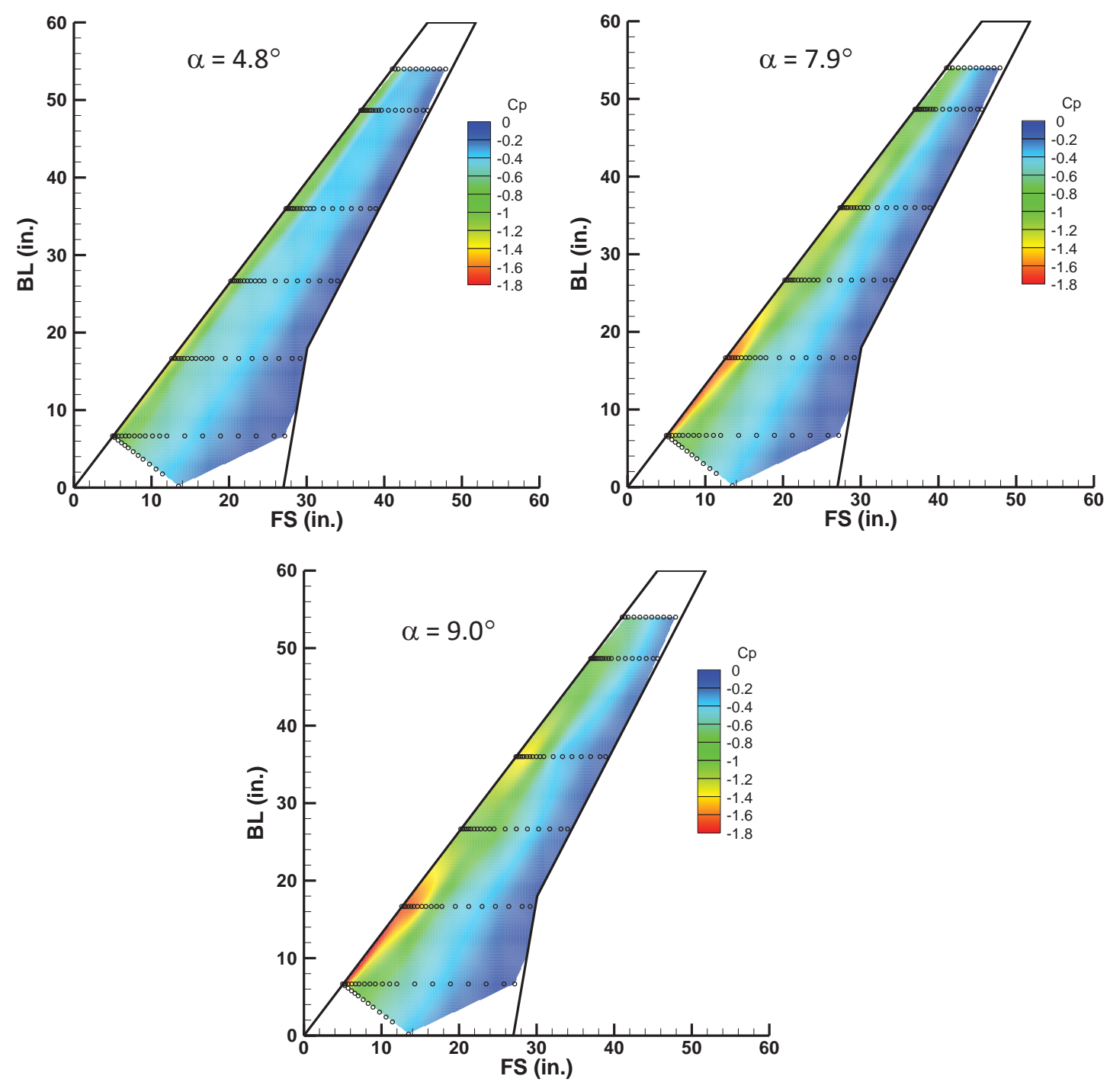

Figure 30: 2D smooth Maximum Scallop configuration pressure contour plots at Re $=1.6 \times 10^{6}$ and $M=0.18$ for $\alpha=4.8^{\circ}, 7.9^{\circ}$, and $9.0^{\circ}$, respectively. ${ }^{17}$

Figure 7 showed that both the 2D smooth and 2D smooth + grit Maximum Scallop configurations had greater maximum lift than the high-fidelity ice shape. Differences in the flowfield between the low- and high-fidelity ice shapes may provide some insight into why this occurred. Features of a leading-edge vortex were visible in Figure 28 and the streamwise vortices observed in the high-fidelity configuration in Figure 24 were not present. As the angle of attack was increased, the area of high suction increased near the leading edge of the wing in Figure 30. Although both the high-fidelity and 2D smooth configurations showed separated flow for the two higher angles of attack in Figure 25 and Figure 29, the 2D smooth configuration showed a much higher leading-edge suction at the highest angle of attack shown here in Figure 30. This higher 
suction most likely caused the higher lift for the 2D smooth Maximum Scallop configuration. The oil-flow visualization, fluorescent mini-tuft visualization, and pressure contour plots for the 2D smooth + grit Maximum Scallop ice shape at $\alpha=4.8^{\circ}, 7.9^{\circ}$, and 8.9 are shown in Figure $31-$ Figure 33. Wake survey data for the 2D smooth + grit Maximum Scallop ice shape at $\alpha=8.9^{\circ}$ is shown in Figure 34.

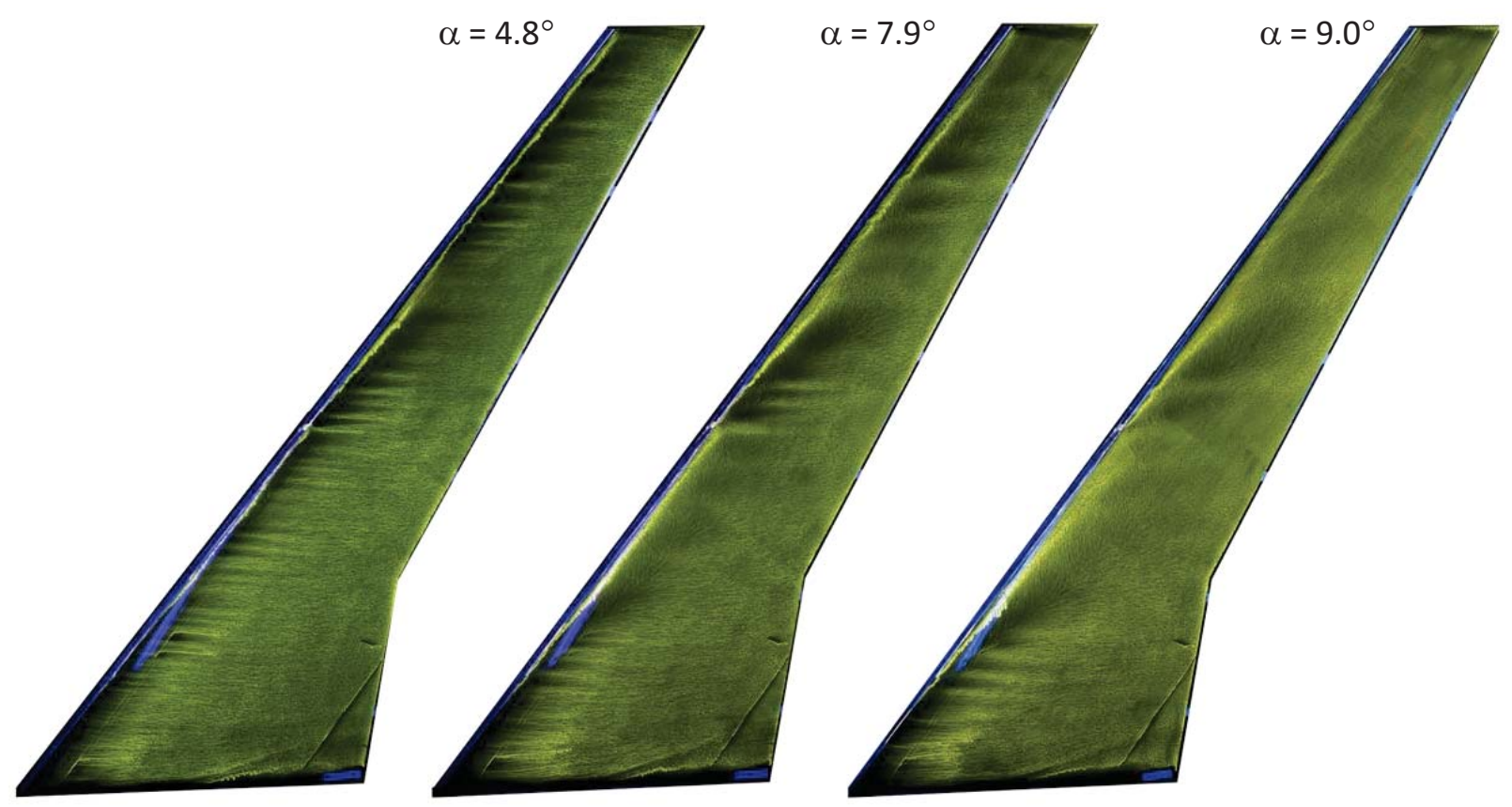

Figure 31: $2 D$ smooth + grit Maximum Scallop configuration oil-flow visualization at $R e=1.6$ $\times 10^{6}$ and $M=0.18$ for $\alpha=4.8^{\circ}, 7.9^{\circ}$, and $9.0^{\circ}$, respectively. ${ }^{17}$ 


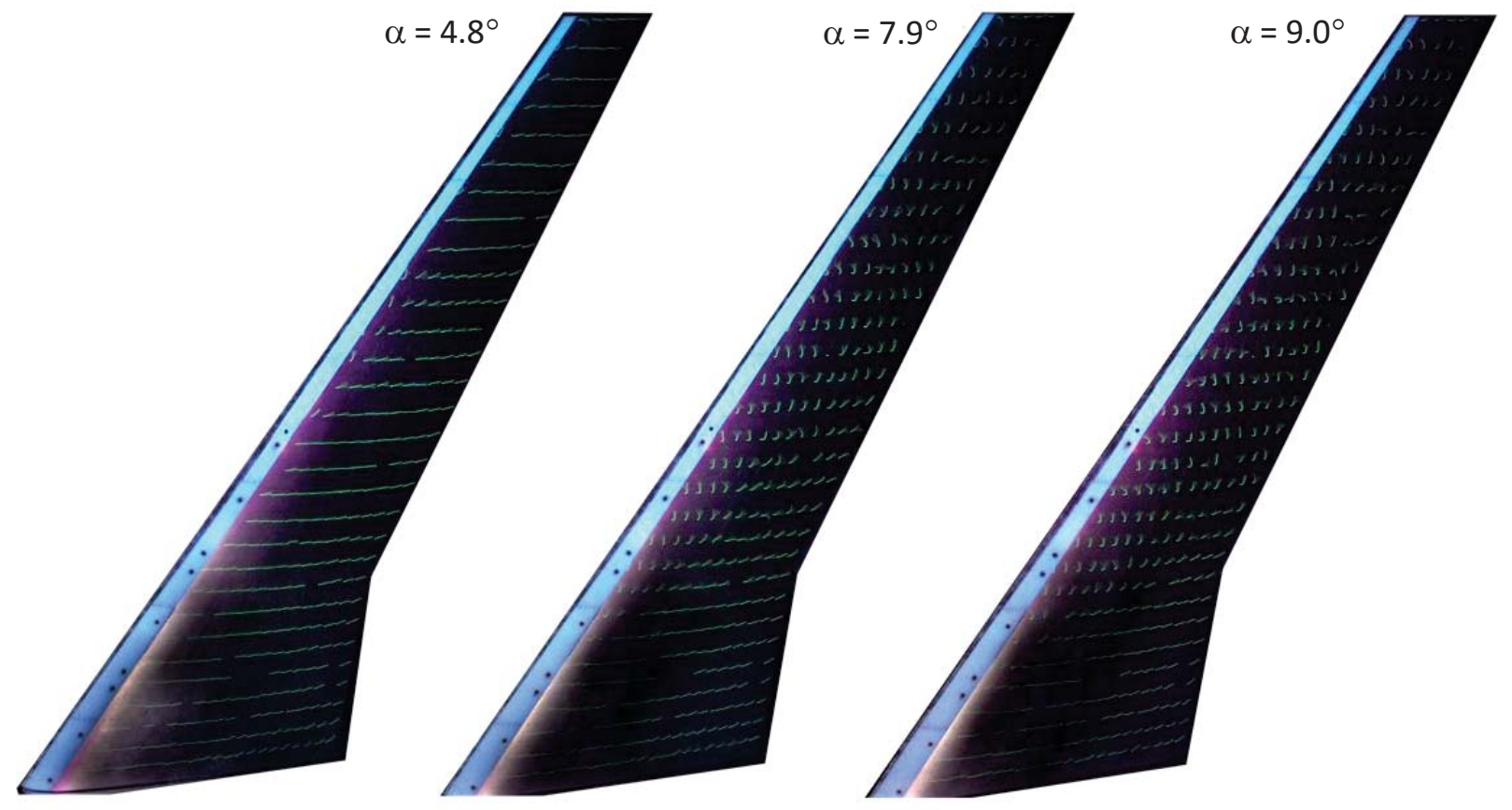

Figure 32: 2D smooth + grit Maximum Scallop configuration fluorescent mini-tuft visualization at $\operatorname{Re}=1.6 \times 10^{6}$ and $M=0.18$ for $\alpha=4.8^{\circ}, 7.9^{\circ}$, and $9.0^{\circ}$, respectively. ${ }^{17}$ 

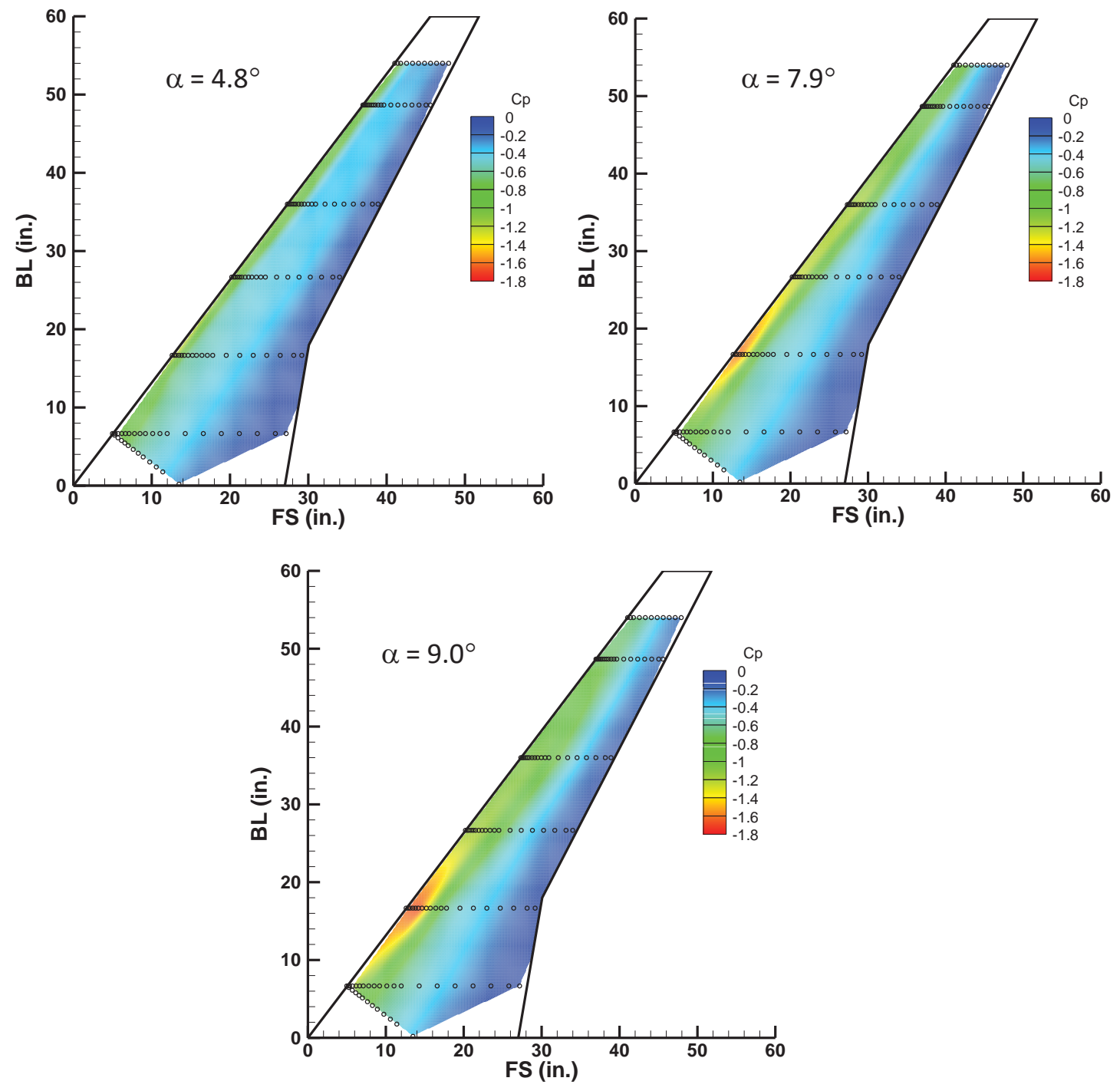

Figure 33: 2D smooth + grit Maximum Scallop configuration pressure contour plots at $R e=1.6$ $\times 10^{6}$ and $M=0.18$ for $\alpha=4.8^{\circ}, 7.9^{\circ}$, and $9.0^{\circ}$, respectively. ${ }^{17}$ 


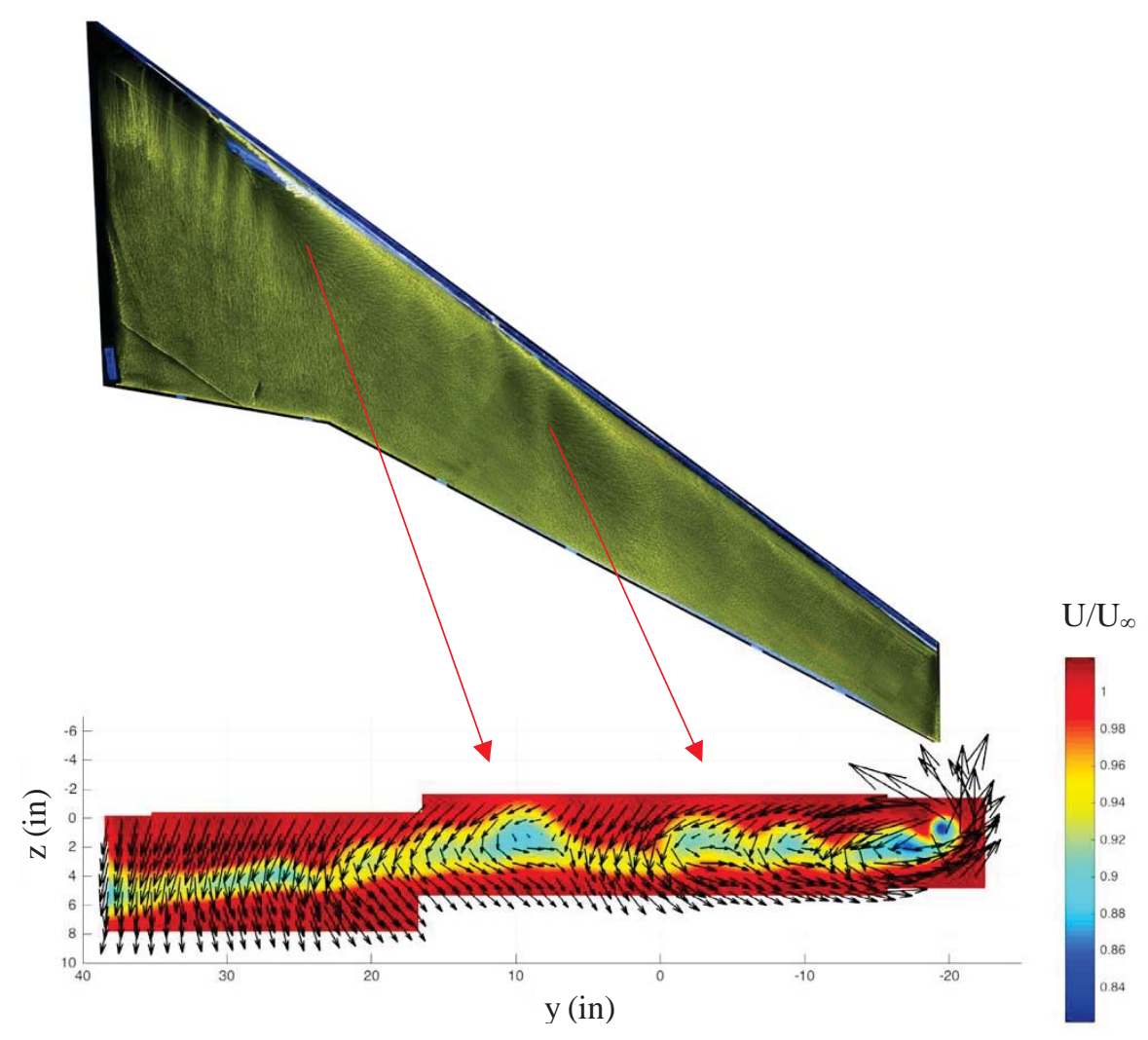

Figure 34: Wake survey velocity plot for 2D smooth + grit Maximum Scallop configuration at Re $=1.6 \times 10^{6}$ and $M=0.18$ for $\alpha=9.0^{\circ}$.

While the oil-flow and mini-tuft visualization showed separation as the angle of attack was increased for all three Maximum Scallop high- and low-fidelity configurations, the biggest differences were in the oil-flow visualization at $\alpha=4.8^{\circ}$ due to the presence of streamwise vortices and pressure contour plots at $\alpha=9.0^{\circ}$. A few streamwise vortices were present at $\alpha=4.8^{\circ}$ in Figure 31, but not as many as the high-fidelity ice shape. This showed that ice shape roughness simulated by grit was in part responsible for the creation of the streamwise vortices. Even with the differences between the oil-flow visualization for all the Maximum Scallop configurations, the roughness did not appear to have a large effect on the 2D smooth Maximum Scallop configuration in the force balance data. This observation is similar to previous results from 2D airfoil tests with horn ice shapes. ${ }^{7}$ In addition, a higher amount of suction was present in the leading-edge area at $\alpha$ $=9.0^{\circ}$ in Figure 33 than the high-fidelity Maximum Scallop configuration in Figure 26 where the leading-edge vortex and streamwise vortices were observed in the oil-flow visualization. Furthermore, the wake survey data for the 2D smooth + grit configuration showed distinct areas 
of separation at $\alpha=9.0^{\circ}$ in Figure 34 while the high-fidelity configuration did not. These differences may have contributed to the differences in aerodynamic performance between the lowand high- fidelity ice shapes.

\section{Conclusions}

Full-span ice shapes of varying geometric fidelity were manufactured for low-Reynolds number wind tunnel testing at the $7 \mathrm{ft} \times 10 \mathrm{ft}$ Walter $\mathrm{H}$. Beech Memorial wind tunnel at Wichita State University. Force balance, surface pressure, and fluorescent mini-tuft visualization data were collected for all ice shape configurations while oil-flow visualization and wake survey data were collected for a subset of the ice shape configurations. Two high-fidelity geometries were chosen for a detailed comparison to two low-fidelity versions of each high-fidelity geometry: a 2D smooth version and a 2D smooth + grit version. The purpose of this paper was to present and compare the preliminary aerodynamic results of ice shapes of varying fidelities from low-Reynolds number tests. Some conclusions are:

- Adding surface roughness to the 2D smooth Streamwise ice shape produced force balance data similar to the high-fidelity Streamwise ice shape force balance data.

○ The 2D smooth + grit Streamwise configuration lift data matched the highfidelity Streamwise configuration lift data almost exactly except at high angles of attack where it produced slightly more lift. This difference in lift production was much smaller than the difference in lift between the 2D smooth + grit and high-fidelity Maximum Scallop configurations.

- The 2D smooth + grit Streamwise configuration drag data matched the highfidelity Streamwise configuration drag data almost exactly except at low angles of attack where it produced slightly more drag. This difference in drag production was much smaller than the difference in drag between the 2D smooth + grit and high-fidelity Maximum Scallop configurations.

- The 2D smooth and 2D smooth + grit Maximum Scallop configurations produced a higher amount of lift compared to the high-fidelity Maximum Scallop configuration, especially at higher angles of attack. 
○ These methods of creating low-fidelity Maximum Scallop ice shapes did not result in a conservative estimate of the lift generated for the high-fidelity Maximum Scallop ice shape geometry.

○ Other methods of creating low-fidelity Maximum Scallop ice shapes may result in force balance data closer to the high-fidelity Maximum Scallop ice shape force balance data.

- Surface pressures on specific regions of the low-Reynolds number swept wing model were similar between some fidelities of the same ice shape geometry. This correlated to similarities between the lift generated between some fidelities of the same ice shape geometry.

○ Creating a low-fidelity ice shape that produced a leading-edge suction similar to the leading-edge suction of a high-fidelity ice shape resulted in lift produced for the low-fidelity ice shape similar to the lift produced by the high-fidelity ice shape.

○ For the Streamwise and Maximum Scallop geometries, the 2D smooth + grit configurations produced force balance data more similar to the high-fidelity force balance data than the 2D smooth force balance data.

- It was observed that the leading-edge suction, and therefore the surface pressure contour plots, for the high-fidelity configurations were more similar to the surface pressure contour plots for the 2D smooth + grit configurations than the surface pressure contour plots for the 2D smooth configurations.

Finally, high-Reynolds number testing will be conducted to study how Reynolds number and Mach number affect the effects of ice shape fidelity on swept wing aerodynamic performance.

\section{References}

\footnotetext{
${ }^{1}$ Broeren, A.P., Potapczuk, M.G., Riley, J.T., Villiedieu, P., Moens, F., and Bragg, M.B., "SweptWing Ice Accretion Characterization and Aerodynamics," AIAA Paper 2013-2824, June 2013, also NASA/TM-2013-216555, Sept. 2013.

2 Broeren, A.P., Addy, H.E., Jr., Bragg, M.B., Busch, G.T., Guffond, D., and Montreuil, E., "Aerodynamic Simulation of Ice Accretion on Airfoils," NASA/TP-2011-216929, June 2011.

${ }^{3}$ Bragg, M.B., Broeren, A.P, and Blumenthal, L.A., "Iced-Airfoil Aerodynamics," Progress in Aerospace Sciences, Vol. 41, July 2005, pp. 323-362.
} 
${ }^{4}$ Broeren, A.P., Bragg, M.B., H.E. Addy, J., Lee, S., Moens, F., and Guffond, D., "Effect of HighFidelity Ice Accretion Simulations on the Performance of a Full-Scale Airfoil Model," AIAA2008-0434, 46 ${ }^{\text {th }}$ AIAA Aerospace Sciences Meeting \& Exhibit, Reno, NV, Jan. 2008, also NASA/TM-2010-216344, June 2010.

${ }^{5}$ Bragg, M.B., Broeren, A.P., Addy, H.E. Jr., Potapczuk, M.G., Guffond, D., Montreuil, E., "Airfoil Ice-Accretion Aerodynamics Simulation," AIAA Paper 2007-0085, January 2008, also NASA TM-2008-214830.

${ }^{6}$ H.E. Addy, J., and Chung, J. J., "A Wind Tunnel Study of Icing Effects on a Natural Laminar Flow Airfoil," AIAA-2000-0095, 38 ${ }^{\text {th }}$ AIAA Aerospace Sciences Meeting \& Exhibit, Reno, NV, Jan. 2000.

${ }^{7}$ Busch, G.T., "Experimental study of full-scale iced-airfoil aerodynamic performance using subscale simulations," Ph.D. thesis, Dept. of Aerospace Eng., Univ. of Illinois, Urbana, IL 2010.

${ }^{8}$ Busch, G., Broeren, A.P., and Bragg, M.B., "Aerodynamic Simulation of a Horn-ice Accretion on a Subscale Model," AIAA-2007-0087, 45 th AIAA Aerospace Sciences Meeting \& Exhibit, Reno, NV, Jan. 2007.

${ }^{9}$ Broeren, A.P., Busch, G., and Bragg, M.B., "Aerodynamic Fidelity of Ice Accretion Simulation on a Subscale Model," SAE 2007 Transactions: Journal of Aerospace, Vol. 116, Aug. 2008, pp. 560-575.

${ }^{10}$ Broeren A.P., Diebold, J.M., and Bragg, M.B., "Aerodynamic Classification of Swept-Wing Ice Accretion," 5th AIAA Atmospheric and Space Environments Conference, San Diego, CA, June 2013, also NASA/TM - 2013-216381, May 2013.

${ }^{11}$ Fujiwara, G. E. C., Wiberg, B. D., Woodard, B., and Bragg, M., "3D Swept Hybrid Wing Design Method for Icing Wind Tunnel Tests", 6th AIAA Atmospheric and Space Environments Conference, AIAA AVIATION Forum, AIAA 2014-2616, June

2014. http://dx.doi.org/10.2514/6.2014-2616

12 Fujiwara, G. E. C., Woodard, B., Wiberg, B., Mortonson, A. J., and Bragg, M., "A Hybrid Airfoil Design Method for Icing Wind Tunnel Tests", 5th AIAA Atmospheric and Space Environments Conference, Fluid Dynamics and Co-located Conferences, AIAA 2013- 2826, June 2013. http://dx.doi.org/10.2514/6.2013-2826

${ }^{13}$ Fujiwara, G.E.C., "Design of 3D swept wing hybrid models for icing wind tunnel tests", M.S. Thesis, Department of Aerospace Engineering, University of Illinois at Urbana-Champaign, 2015.

${ }^{14}$ Broeren, A.P., Potapczuk, M.G., Lee, S., Malone, A.M., Paul, B.P., Jr., and Woodard, B.S., "IceAccretion Test Results for Three Large-Scale Swept-Wing Models in the NASA Icing Research Tunnel," AIAA Paper 2016-3733, June 2016, also NASA/TM-2016-219137, Sept. 2016.

${ }^{15}$ Camello, S.C., Lee, S., Lum, C., and Bragg, M.B., "Generation of Fullspan Leading-Edge 3D Ice Shapes for Swept-Wing Aerodynamic Testing," AIAA 8th Atmospheric and Space Environments Conference, Washington D.C., June 13-17, 2016, AIAA Paper 2016-3737.

16 Broeren, A.P., Woodard. B.S., Diebold, J.M., and Moens, F., "Low-Reynolds Number Aerodynamics of an 8.9\% Scale Semispan Swept Wing for Assessment of Icing Effects," AIAA $9^{\text {th }}$ Atmospheric and Space Environments Conference, Denver, CO, June 5-9, 2017 (submitted for publication), 2017.

${ }^{17}$ Camello, S.C., "Effect of Ice Accretion Simulation Fidelity on Low-Reynolds Number SweptWing Aerodynamic Performance," Masters Thesis, Dept. of Aeronautics \& Astronautics, Univ. of Washington, Seattle, WA, 2017. 
18 "Data Reduction System: 3D Reflection Plane Coordinate System Definition, Moment Transfers, Coordinate Rotations," Walter H. Beech Wind Tunnel Engineering Process Description, Rev. A., Apr. 2012.

${ }^{19}$ Pope, A., Rae, W.H., and Barlow, J.B., "Low-Speed Wind Tunnel Testing," John Wiley \& Sons, Inc., 3rd ed., 1999.

${ }^{20}$ Diebold, J.M., Woodard, B.S., Camello, S.C., Bragg, M.B., "Effect of Ice Accretion of FullScale, Swept-Wing, Aerodynamic Performance and Control Effects," DOT/FAA/TC-17/22 (to be published)

${ }^{21}$ Vassberg, J.C., DeHann, M.A., Rivers, S.M., and Wahls, R.A., "Development of a Common Research Model for Applied CFD Validation Studies," AIAA Paper 2008-6919, Aug. 2008.

${ }^{22}$ Kaszycki, M., Federal Aviation Administration, "Performance and Handling Characteristics in Icing Conditions" (Advisory Circular 25-25A), 2014.

23 "Product Data Sheet: Somos ${ }^{\circledR}$ NeXt," Somos ${ }^{\circledR}$, URL: https://www.dsm.com/

24 Lum, C.W., Sandhu, N., Diebold, J.M., Woodard, B.S., and Bragg, M.B., "The Application of a Five-Hole Probe Wake-Survey Technique to the Study of Swept Wing Icing Aerodynamics," AIAA $9^{\text {th }}$ Atmospheric and Space Environments Conference, Denver, CO, June 5-9, 2017 (submitted for publication), 2017. 